\title{
USING MONOCULAR VISION AND IMAGE CORRELATION TO ACCOMPLISH AUTONOMOUS LOCALIZATION
}

\author{
A Thesis \\ Presented to the \\ Faculty of California Polytechnic State University \\ San Luis Obispo
}

\author{
In Partial Fulfillment \\ of the Requirements for the Degree \\ Masters of Science in Computer Science
}

By

Matthew P. Schlachtman

June 2010 
(C) 2010

Matthew P. Schlachtman

ALL RIGHTS RESERVED 


\section{COMMITTEEE MEMBERSHIP}

TITLE: Monocular Vision and Image Correlation to Accomplish Autonomous Localization

AUTHOR: Matthew P. Schlachtman

DATE SUBMITTED: June 2010

COMMITTEE CHAIR: Christopher M. Clark, Ph.D.

COMMITTEE MEMBER: Franz Kurfess, Ph.D.

COMMITTEE MEMBER: Saeed Niku, Ph.D. 


\begin{abstract}
Monocular Vision and Image Correlation to Accomplish Autonomous Localization Matthew P. Schlachtman
\end{abstract}

For autonomous navigation, robots and vehicles must have accurate estimates of their current state (i.e. location and orientation) within an inertial coordinate frame. If a map is given a priori, the process of determining this state is known as localization. When operating in the outdoors, localization is often assumed to be a solved problem when GPS measurements are available. However, in urban canyons and other areas where GPS accuracy is decreased, additional techniques with other sensors and filtering are required.

This thesis aims to provide one such technique based on monocular vision. First, the system requires a map be generated, which consists of a set of geo-referenced video images. This map is generated offline before autonomous navigation is required. When an autonomous vehicle is later deployed, it will be equipped with an on-board camera. As the vehicle moves and obtains images, it will be able to compare its current images with images from the pre-generated map. To conduct this comparison, a method known as image correlation, developed at Johns Hopkins University by Rob Thompson, Daniel Gianola and Christopher Eberl, is used. The output from this comparison is used within a particle filter to provide an estimate of vehicle location. Experimentation demonstrates the particle filter's ability to successfully localize the vehicle within a small map that consists of a short section of road. Notably, no initial assumption of vehicle location within this map is required. 


\section{Contents}

List of Figures

1 INTRODUCTION

2 BACKGROUND $\quad 2$

3 PROBLEM DEFINITION \& SOLUTION 9

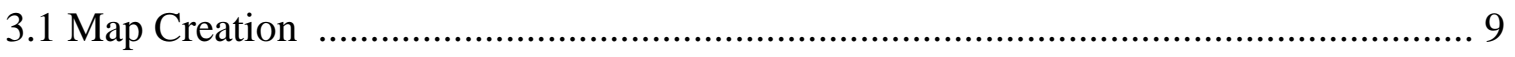

3.1.1 Video Capture ............................................................................ 11

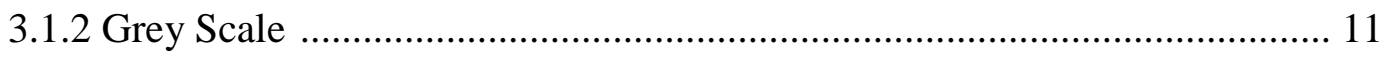

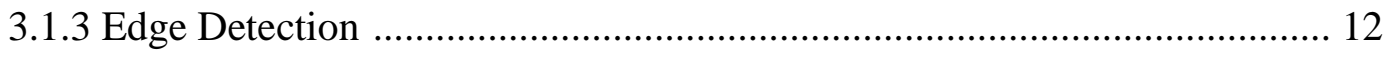

3.1.5 Map Image Selection ................................................................. 13

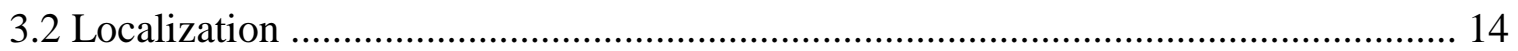

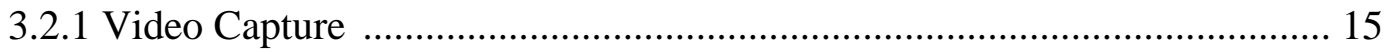

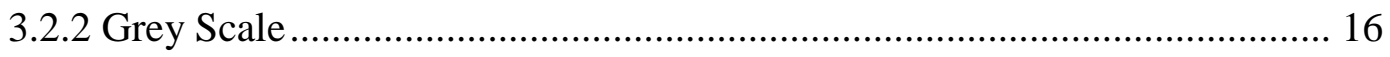

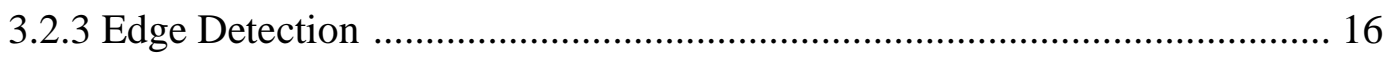

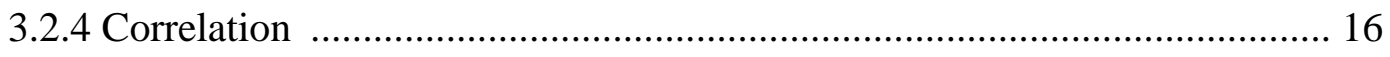

3.2.6 Map Image Match Determination ...................................................... 16

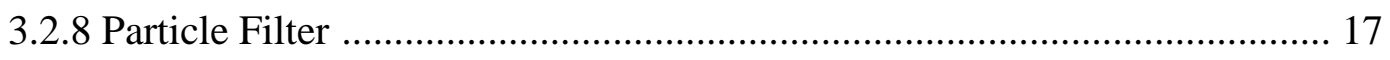

\section{IMPLEMENTATION}

4.1 Implementation of Map Creation ...................................................................

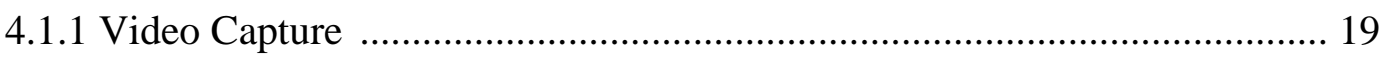

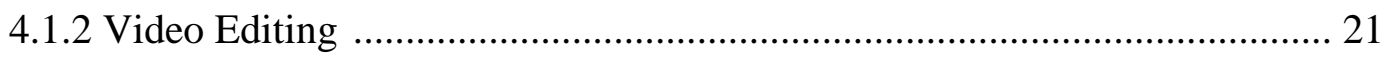

4.1.3 Grey Scale \& Edge Detection ......................................................... 22

4.1.4 Converting Video Format ............................................................... 24 


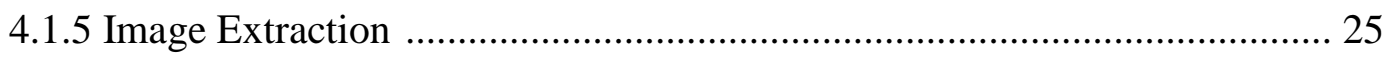

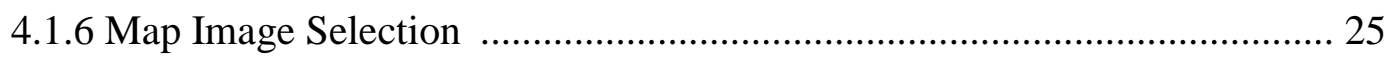

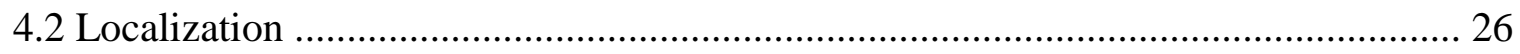

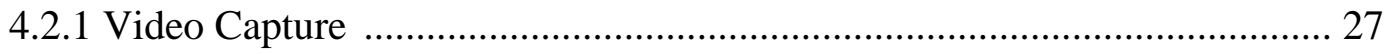

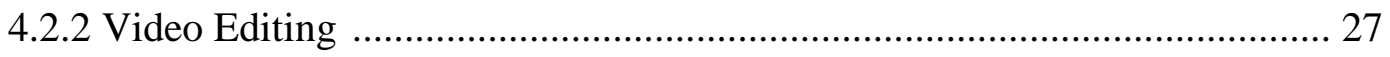

4.2.3 Grey Scale \& Edge Detection ........................................................ 28

4.2.4 Converting Video Format .............................................................. 28

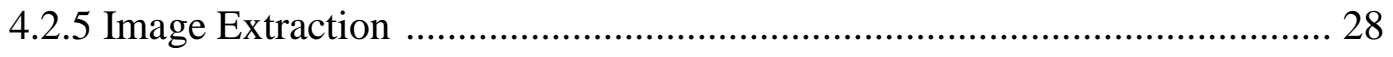

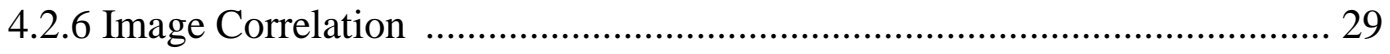

4.2.7 Map Image Match Determination .................................................. 34

4.2.8 Particle Filter .................................................................................. 35

5 RESULTS $\quad 36$

6 CONCLUSION

7 FUTURE WORK \& POTENTIAL APPLICATIONS 42

$\begin{array}{ll}\text { Bibliography } & 43\end{array}$

Appendix A: 'smooth_try3.m' 45

Appendix B: 'IP_PF_0.m' 46 


\section{List of Figures}

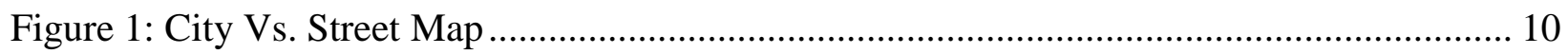

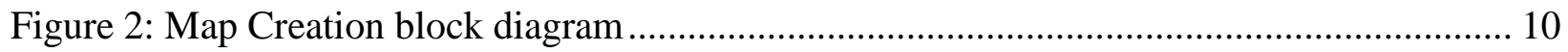

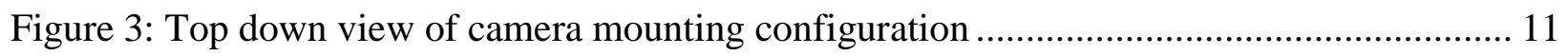

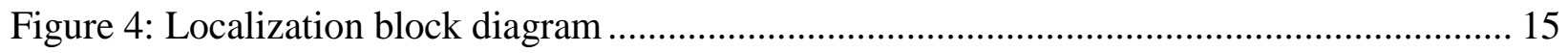

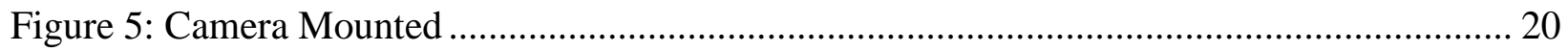

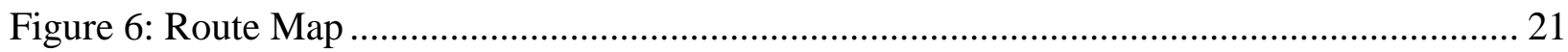

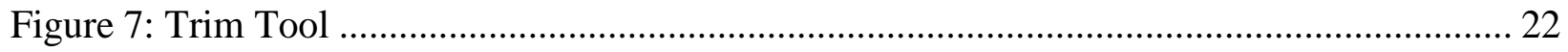

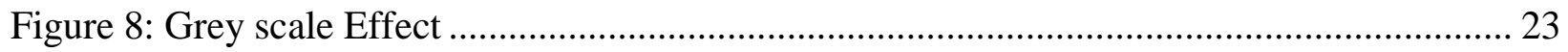

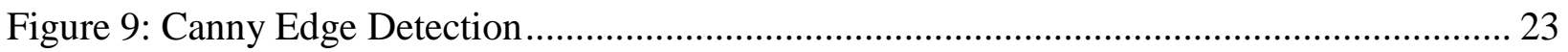

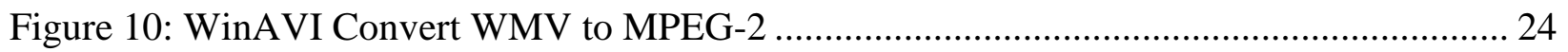

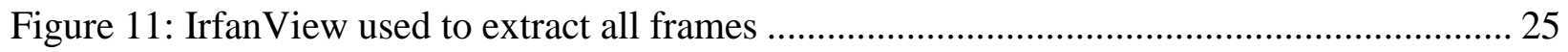

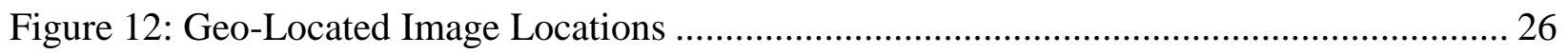

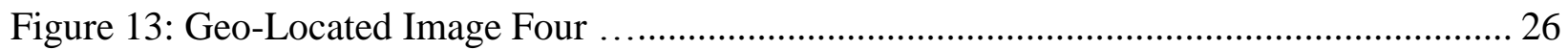

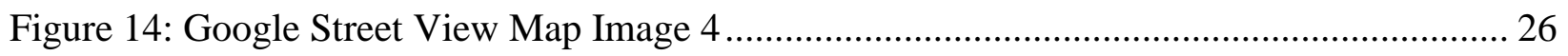

Figure 15: Start of Driving Run ......................................................................................... 27

Figure 16: Visual Compare, 3rd Geo-located frame on left, Frame 587 from Localization

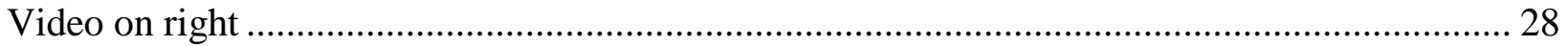

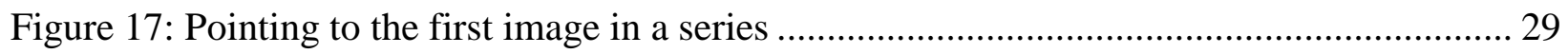

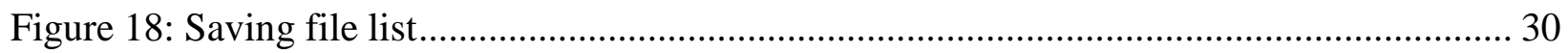

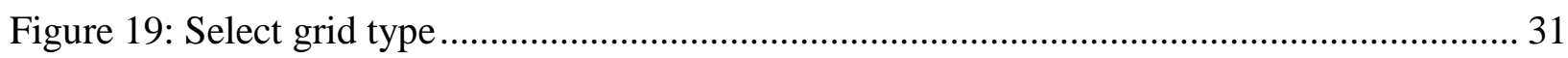

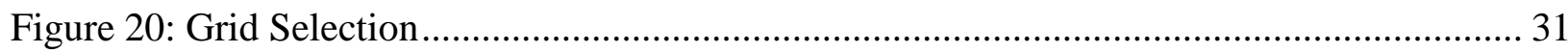

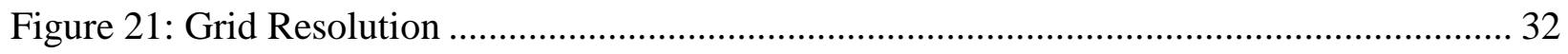

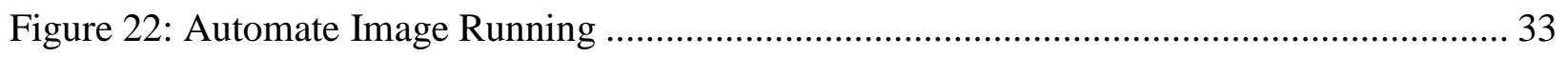

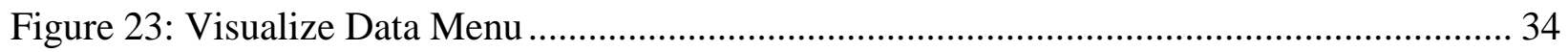

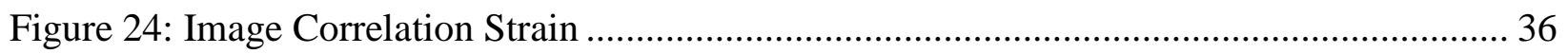

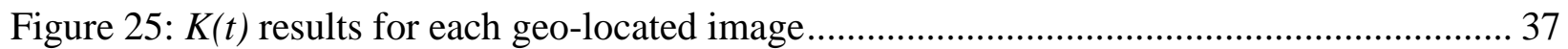

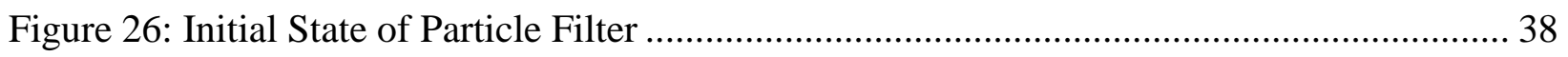

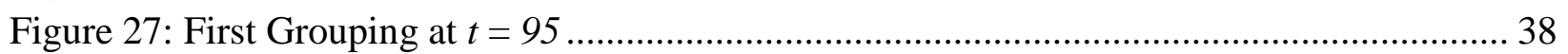

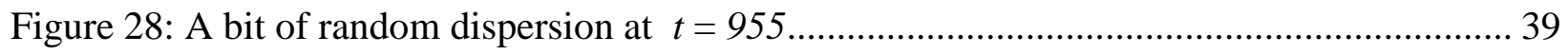

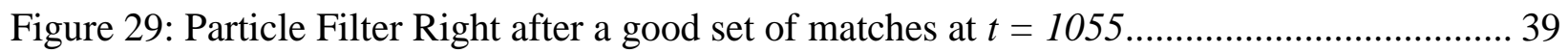

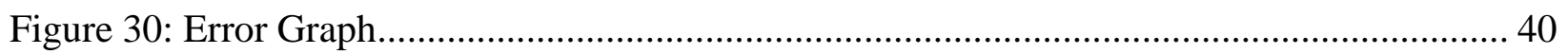




\section{Chapter 1}

\section{INTRODUCTION}

One of the design objectives of computer vision localization is to provide a low cost method for outdoor localization using a single camera. [3] This relaxes the need for global positioning system (GPS), which may experience degraded reliability in urban settings like in downtown settings with more tall buildings that could block the signals from the GPS satellites from getting to the GPS receiver. It is possible to imagine TomTom or Garmin, both commercial GPS manufacturers, implementing a small camera on the GPS system that could be looking out in front of the car and perform the kind of visual localization that has been discussed. Moreover, this type of localization could be useful in the operation of future autonomous vehicles.

The goal of this thesis is to demonstrate that by using a combination of existing technology, autonomous localization can be accomplished with a single camera obtaining images of the view outside of the car. This thesis intends on demonstrating that through the use of image processing, edge detection, and image correlation and comparison, one image can be compared with another, image from a database of geo-located images to determine the location of a vehicle. While only a proof of concept, the idea could be expanded on and can be adapted to serve real world purposes and potentiality a profitable idea or product. 


\section{Chapter 2}

\section{BACKGROUND}

Localization is the act of determining the location of an (autonomous) agent within a map given a priori. In almost every type of mobile robot system localization is necessary. Regardless of the size of the robot, the system will most likely need to know where it or something else is. Various aspects to robotics count on this ability, including but not limited to, robotic navigation. Success in navigation requires success at the four building blocks of navigation:

- Perception - the robot must interpret its sensors to extract meaningful data from its environment

- Localization - the robot must determine its position in the environment, or the position of some element of interest in the environment.

- Cognition - the robot must decide how to act to achieve its goals.

- Motion Control - to robot must modulate its motor outputs or other actuators to achieve the desired trajectory [2].

Autonomous vehicle localization can be accomplished with various different hardware and software components. There are several different families of hardware that can be used to accomplish localization. There are also algorithms to facilitate the localization using the various localization hardware types [1]. Some of the most popular are Markov Localization, Particle Filter Localization, and Kalman Filter Localization [1].

Markov localization uses an explicitly specified probability distribution across all possible robot positions [2]. Instead of maintaining a single hypothesis as to where in the world a robot might be, Markov localization maintains a probability distribution over the space of all such hypotheses. The probabilistic representation allows it to weigh these different hypotheses in a mathematically sound way [10]. For the sake of simplicity, let's assume that the space of robot positions is one-dimensional, that is, the robot can only move horizontally (it may not rotate). Now suppose the robot is placed somewhere in this environment, but it is not told its location. 
Markov localization represents this state of uncertainty by a uniform distribution over all positions. Now let's assume the robot queries its sensors and finds out that it is next to a door. Markov localization can modify the belief by raising the probability for places next to doors, and lowering it anywhere else. Now let's assume the robot moves a meter forward. Markov localization incorporates this information by shifting the belief distribution accordingly. To account for the inherent noise in robot motion, which inevitably leads to a loss of information, the new belief more evenly distributes likelihoods across all robot states (and less certain) than the previous one. Finally, let's assume the robot senses a second time, and again it detects another door. At this point, the belief is updated to again increase likelihoods of all states next to the doors [10] [11].

The particle filter is an implementation of the Baye's filter using a finite number of particles in the continuous state space to describe the belief state probability density distribution. The algorithm propagates particles through time using the survival of the fittest concept [3]. The main objective of particle filtering is to track a variable or interest as it evolves over time, typically with a non-Gaussian and potentially multi-modal probability density function. The basics of the method are to construct a sample-based representation of the entire probability density function. A series of actions are taken, each one modifying the state of the variable of interest according to some model. Moreover, certain observations can constrain the state of the variable of interest at that time [9]. Multiple particles of the variable of interest are used, each one with an assigned weight that signifies the likelihood of that specific particle. An estimate of the variable of interest can be obtained by the weighted sum of all of the particles. Like most localizations algorithms, the particle filter algorithm is recursive in nature and operates in two phases; prediction and correction. After each action, each particle is modified according to the existing model (prediction stage), including the addition of random noise in order to simulate the uncertainty in the model. Then, each of the particle's weight is evaluated based on the latest sensory information available (update/correction stage). Particles with high weights have a higher likelihood of remaining when the resampling occurs [9]. 
Kalman Filter Localization, another commonly used approach, uses a Gaussian probability density representation of robot position [2]. The Kalman filter iterates over the consecutive cycles of prediction and correction. The dynamics of these cycles is derived and interpreted in the framework of Gaussian probability density functions. Under additional conditions on the system dynamics, the Kalman filter dynamics converge to a steady-state filter and the steady-state gain is derived. The innovation process associated with the filter, that represents the novel information conveyed to the state estimate by the last system measurement, is introduced. The states estimates are interpreted in terms of the covariance associated with the Gaussian probability density function involved in the filter dynamics [12].

All of the localization algorithms, including those mentioned above, are able to be set up in such a way that they can be adapted with various motion and sensor models to correctly implement any particular mode of locomotion and sensing. Picking the algorithm is usually based on the actual hardware, system, and environment. Once one knows the requirements for a project the optimal algorithm can be chosen [1].

Sonar (originally an acronym for sound navigation and ranging) is a technique that uses sound propagation (usually underwater) to navigate, communicate with or detects other vessels. There are two kinds of sonar: active and passive. Sonar may be used as a means of acoustic location and of measurement of the echo characteristics of "targets" in the water. Acoustic location in air was used before the introduction of radar. Sonar may also be used in air for robot navigation, and SODAR (an upward looking in-air sonar) is used for atmospheric investigations. The term sonar is also used for the equipment used to generate and receive the sound. The frequencies used in sonar systems vary from infrasonic to ultrasonic. The study of underwater sound is known as underwater acoustics or sometimes hydro acoustics [13]. Sonar is a good source for localization but usually in smaller environments and can be interfered with by many things that emit sound in nature. Sonar works best for localization when applied to underwater robots. In fact, the VideoRay ROV robot uses sonar and this robot has been used quite a bit to create maps and perform SLAM algorithms (Simultaneous Localization and Mapping) [5]. As well this technology is the primary sensor for the Malta Cistern Mapping project founded by Dr. Christopher Clark [14]. 
Infrared Range sensors are commonly found on smaller robots that are used for exploring places like buildings, caves, and other such relatively small environments. These sensors work on the premise that invisible (to humans) light is emitted by aED, bounces off a surface, and is perceived by Infrared photocells. The time that it takes for the light to travel, bounce off of something, and travel back to the photocell can be used to calculate the distance that the light hit something at. This type of sensing technology is likely to be the least expensive but is also very unreliable. The technology is subject to errors caused by simple room lights, the sun, and various electronic devices. The problem is that the photocell may not be able to differentiate between the light the LED emits and various other sources in the environment [2].

A laser rangefinder is a device that uses a laser beam in order to determine the distance to a reflective object. The most common form of laser rangefinder operates on the time of flight principle by sending a laser pulse in a narrow beam towards the object and measuring the time taken by the pulse to be reflected off the target and returned to the sender. Due to the high speed of light, this technique is not appropriate for high precision sub-millimeter measurements, where triangulation and other techniques are often used [13]. Although Laser Scanners are very accurate; their cost is very high. Even the simplest models go for thousands of dollars [15]. As well, the technology is not really the best sensor for mapping, and its localization ability is based of past data or some type of three-dimensional map [1].

GPS (Global Positioning System) works by having a network of twenty-four satellites in orbit around earth. Global Positioning System satellites transmit signals to equipment on the ground. GPS receivers passively receive satellite signals; they do not transmit. GPS receivers require an unobstructed view of the sky, so they are used only outdoors and they often do not perform well within forested areas or near tall buildings. GPS operations depend on a very accurate time reference, which is provided by atomic clocks at the U.S. Naval Observatory. Each GPS satellite has atomic clocks on board [16]. Each GPS satellite transmits data that indicates its location and the current time. All GPS satellites synchronize operations so that these repeating signals are transmitted at the same instant. The signals, moving at the speed of light, arrive at a GPS receiver at slightly different times because some satellites are farther away than others. The 
distance to the GPS satellites can be determined by estimating the amount of time it takes for their signals to reach the receiver. When the receiver estimates the distance to at least four GPS satellites, it can calculate its position in three dimensions [16].

In current technology, GPS is seen as being one of the best ways to localize one's position [2]. With accuracy down to a few meters, and costs in the 100 to 200 dollar range it is no wonder it's used so heavily. The accuracy of a position determined with GPS depends on the type of receiver. Most hand-held GPS units have about ten to twenty meter accuracy. Other types of receivers use a method called Differential GPS (DGPS) to obtain much higher accuracy. DGPS requires an additional receiver fixed at a known location nearby. Observations made by the stationary receiver are used to correct positions recorded by the roving units, producing accuracy greater than 1 meter [16]. When the system was created, timing errors were inserted into GPS transmissions to limit the accuracy of non-military GPS receivers to about 100 meters. This part of GPS operations, called Selective Availability, was eliminated in May 2000. If one could attach an accurate GPS sensor to a mobile robot, much of the localization problem would be obviated [16].

The GPS would inform the robot of its exact position, so the answer to the question “Where Am I?" would always be available immediately. Unfortunately, such a sensor is not always practical. The existing GPS network provides accuracy to within several meters, which may be unacceptable for localizing on much smaller areas. Furthermore, GPS technology cannot function indoors or in obstructed areas and are thus limited in their workspace. [2] In large city areas there can sometimes be issues when localizing using GPS due to the fact that the GPS signals can be impeded or blocked completely by buildings that get in the way of the line of sight which is needed for GPS to function properly. Hypothetically speaking, an alternate sensor payload could be used in conjunction with GPS to help with the localization when GPS signals become unreliable or nonexistent.

There are other solutions besides GPS that are very useful and have various benefits that GPS can't match. Various small solutions include sonar and infrared distance sensors, cameras, and even laser range finders and scanners [4]. The intended are of focus with the aforementioned 
area studies, is the use of monocular computer vision. This type of vision would be a great alternative for localization when convention GPS has failed due to any number of circumstances. Other researchers have also used monocular vision in localization because of the simplicity of hardware involvement.

It is possible that computer vision could be a good alternative or supplement to GPS. In recent publications, researchers have tried using a database of images to serve as the map in localization. Zhang et al. [4] captured images at various points in an operating workspace and tagged them with GPS position readings to create an image database [3]. Similar work has also been done by looking at the details of building facades. In the most related and recent work presented in [7], a robot is first guided through a course as it records a video of the surrounding. This information is used offline where distinct image features are selected to generate a three dimensional map [3].

Using computer vision and edge detection a reference can be made to detect location based on images or edges detected compared to another image and set of edges in a stored database. The first step in obtaining a feature map is the processing of raw image data using edge detector to create an edge map which highlights the pixels that are likely to be part of building boundaries [3]. A comparison of the edges detected to pre processed images yields a probability distribution that would be associated with all possible locations the robot could be in within the environment. [2].

The orientations of observed building walls need to be interpreted from camera images. This information can be recovered from the effect of perspective using vanishing point analysis [7]. A benefit of using vanishing point analysis and selecting building orientation as a measure for comparison is that it makes the system tolerant to significant camera rolling and tilting. Edge detection is used to extract useful features from an image scene [3].

There exists a free library for computer vision known as OpenCV, which has the ability to implement the computer vision procedures mentioned above. OpenCV is a computer vision library originally developed by Intel. It is free for commercial and research use under a BSD 
license. The library is cross-platform, and runs on Windows, Mac OS X, Linux, PSP, VCRT (Real-Time OS on Smart camera) and other embedded devices. It focuses mainly on real-time image processing, as such, if it finds Intel's Integrated Performance Primitives on the system, it will use these commercial optimized routines to accelerate itself [13][17].

Many computer vision techniques have also been programmed into the math simulator known as Matlab. MATLAB stands for "MATrix LABoratory" and is a numerical computing environment and fourth-generation programming language. Developed by The MathWorks, MATLAB allows matrix manipulations, plotting of functions and data, implementation of algorithms, creation of user interfaces, and interfacing with programs written in other languages, including C, C++, and FORTRAN [18]. Unlike the OpenCV libray, the matlab image processing and computer vision libraries enable a user to focus more on the theory of the project and less on the computer programming and syntax of the language, as using MATLAB is a very simple tool for performing simulations, and requires less programming skill. While MATLAB is not primarily used for computer vision applications, in more recent builds, MATLAB has been including various computer vision and image processing libraries allowing the necessary mathematical operation to function over videos and images [19].

There is a MATLAB library called Digital Image Correlation and Tracking posted by Christopher Eberl, which was of particular interest for the purpose of this paper as it is the primary method of which image correlation is accomplished in the experiments talked about in the latter sections. The Library's given description is that is calculates displacement and strain from a series of images. Strain can be calculated in horizontal as well as vertical direction. The functions were developed at the Johns Hopkins University by Rob Thompson, Daniel Gianola and Christopher Eberl. The code is a continued work in progress and was originally released in September of 2006, and was most recently updated in September of 2008. The code was specifically designed to either produce the calculations necessary for image correlation, or to assist in a form of feature tracking. For the cases of this thesis, correlation section of the library will be used. 


\section{Chapter 3}

\section{PROBLEM DEFINITION \& SOLUTION}

The problem addressed in this thesis is defined as:

Given a previously recorded video set parameterized within a geolocalized coordinate system, determine the geo-referenced location of a vehicle using only an on-board monocular vision system.

The proposed solution to this problem can be broken into two basic components: map creation and localization. This section sets the scene for building the low level detail upon how each one of the tasks should be accomplished. The implementation chapter on the other hand, goes into the technical details of the experiment.

\subsection{Map Creation}

A map must be created with geo-located points that correspond to specific frames in a pre-recorded video such that:

$$
\begin{gathered}
V_{m}=>M \\
M=\left\{\left[I_{m 1}, x_{m 1}, y_{m 1}\right],\left[I_{m 2}, x_{m 2}, y_{m 2}\right], \ldots,\left[I_{m n}, x_{m n}, y_{m n}\right]\right\}
\end{gathered}
$$

The above equations symbolize the goal of the map creation part of the Solution. In equation (1) and (2), $M$ refers to the map itself, $V_{m}$ refers to the prerecorded video. $I_{m l}$ refers to a geo-located image frame from the video such that $x_{m l}$ and $y_{m l}$ represent the corrdinates on the map. The following flow chart explains the necessary steps that need to be taken to construct the map. 


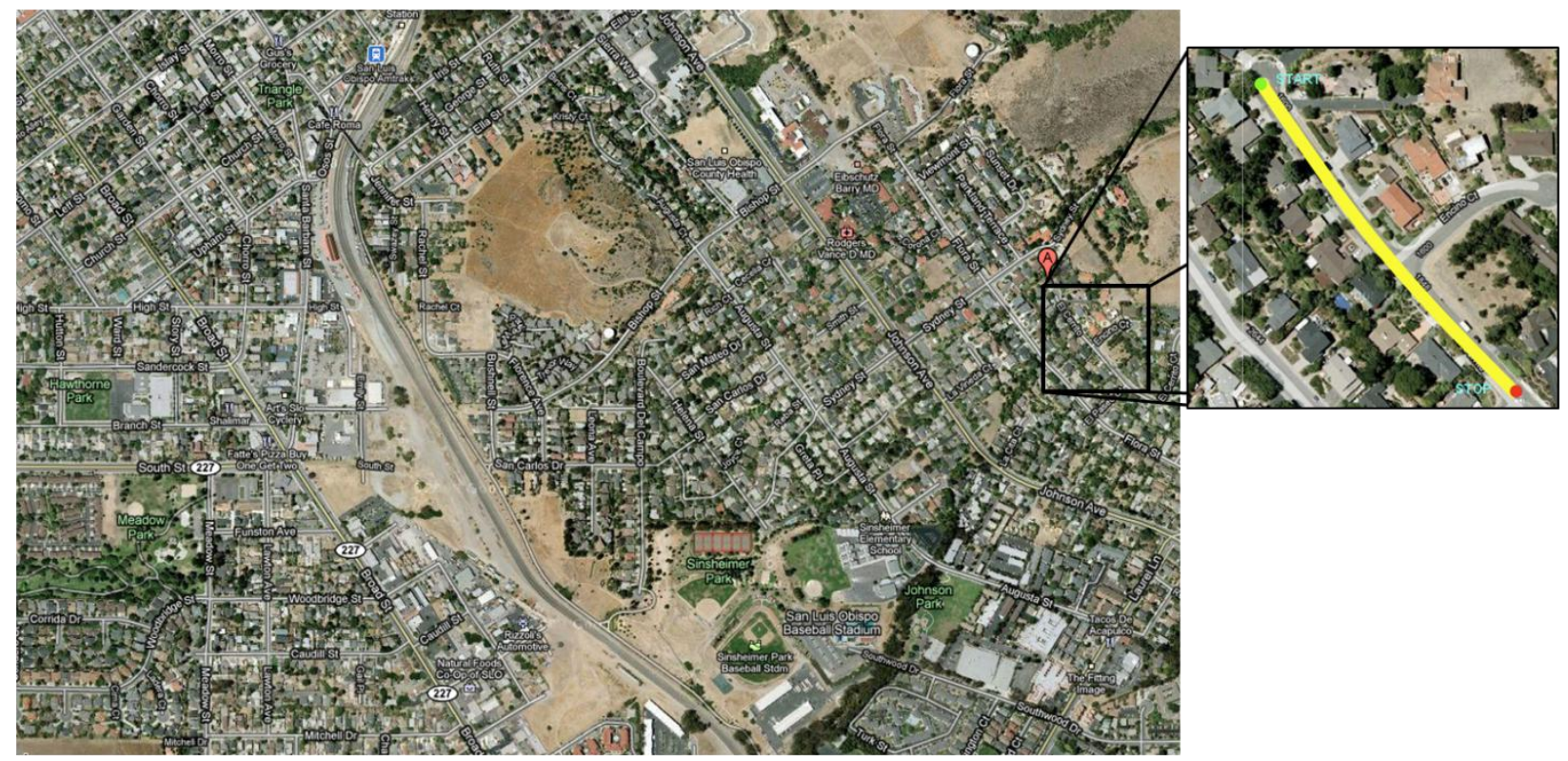

Figure 1: City Vs. Street Map

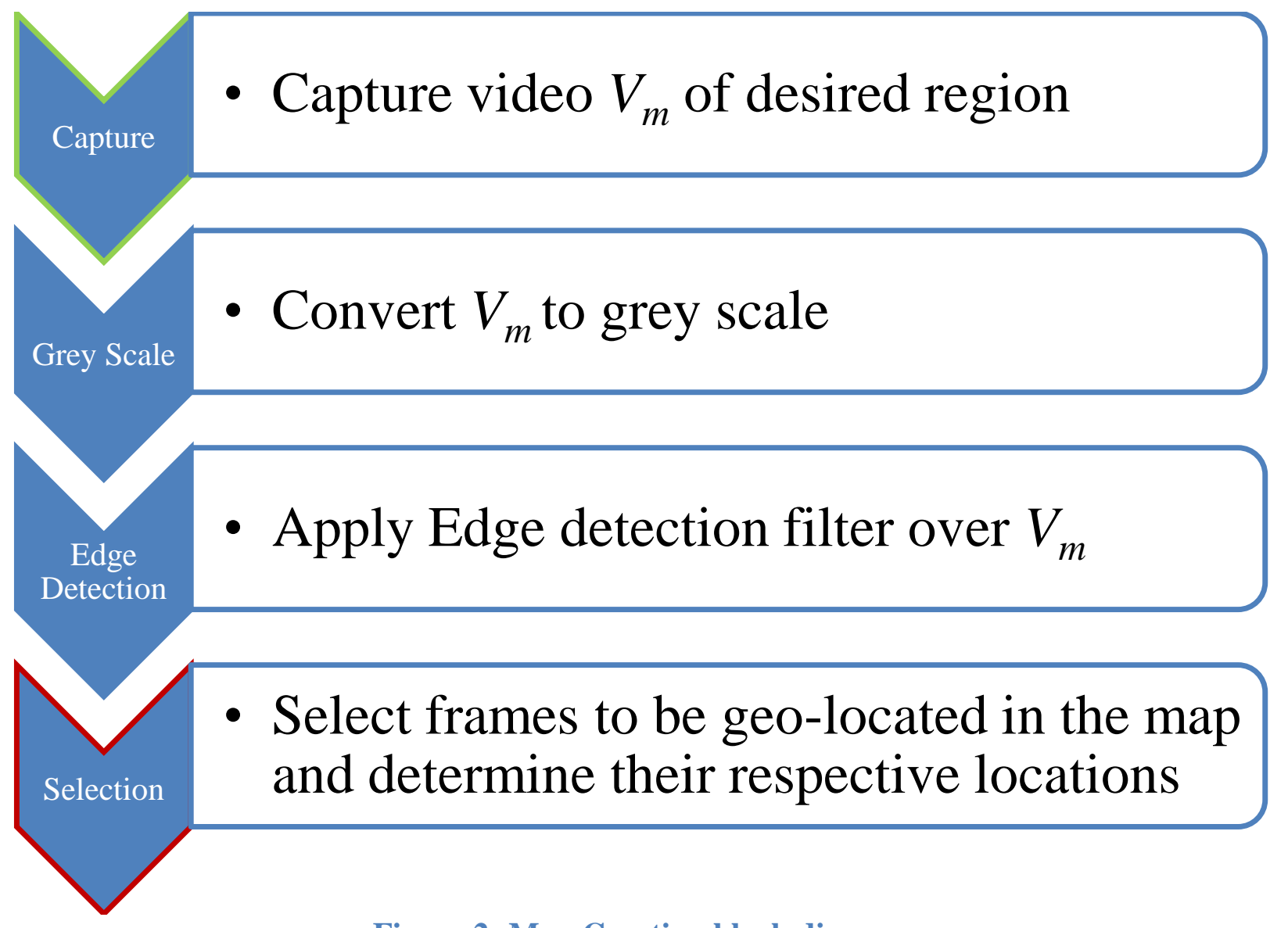

Figure 2: Map Creation block diagram 
The above block diagram (figure 2) demonstrates how the map creation process is split into four phases: Capture, Grey Scale, Edge Detection, and Selection.

\subsubsection{Video Capture}

The first step in constructing a map is obtaining a video $V_{m}$. Video is recorded while the vehicle is driven down a length of road. Camera configuration should capture the view outside of the passenger side window as shown in Figure 3 below.

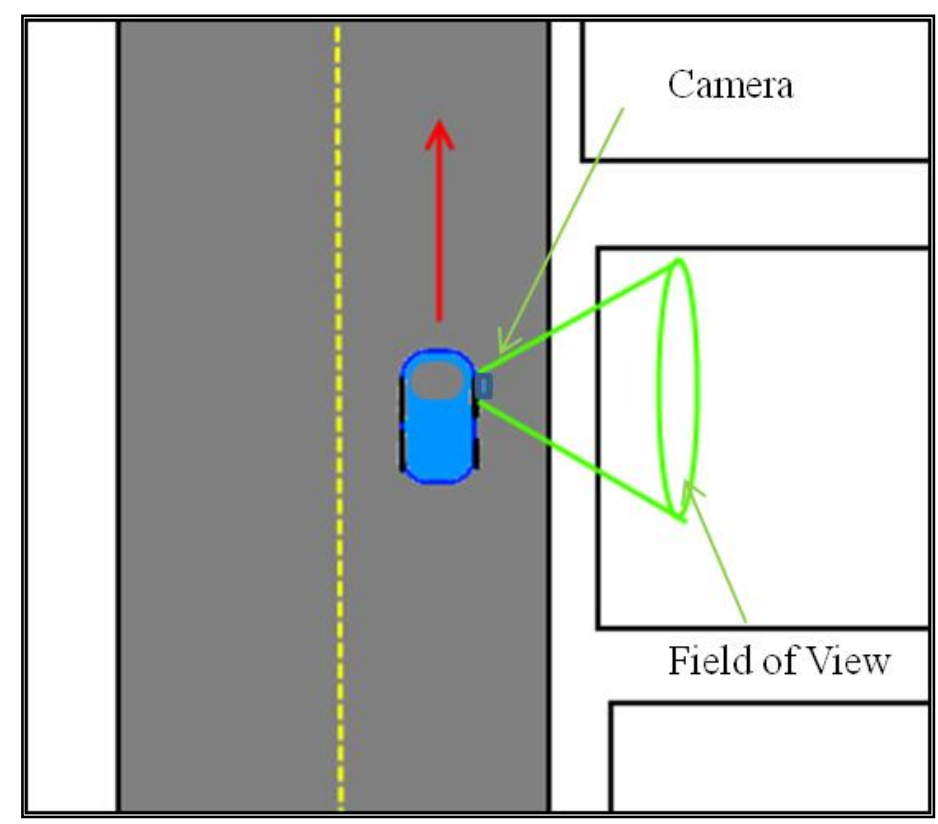

Figure 3: Top down view of camera mounting configuration

\subsubsection{Grey Scale}

Computer vision and/or video processing tools are used to convert the videos to grey scale. Conversion of a color image to grey scale is not unique; different weighting of the color channels effectively represents the effect of shooting black-and-white film with different-colored photographic filters on the cameras. A common strategy is to match the luminance of the grey scale image to the luminance of the color image. To convert any color to a grey scale representation of its luminance, first one must obtain the values of its red, green, and blue (RGB) primaries in linear intensity encoding, by gamma expansion. Then, add together $30 \%$ of the red 
value, $59 \%$ of the green value, and $11 \%$ of the blue value (these weights depend on the exact choice of the RGB primaries, but are typical), as seen in (3). The resultant number is the desired linear luminance value which is applied to a white to black scale [22].

$$
\begin{aligned}
g_{i j}=0.30 R_{i j}+0.59 G_{i j}+0.11 B_{i j} \text { for } i & =0 \ldots . \text { Image Width } \\
j & =1 \ldots \text { Image Height }
\end{aligned}
$$

\subsubsection{Edge Detection}

Using computer vision and/or video processing tools a Canny edge detection filter is applied over all of the frames of the video. The grey scale is applied first as it is typically easier to apply an edge detection filter when dealing with a single intensity value (i.e. Black). The Canny edge detector uses a filter based on the first derivative of a Gaussian, because it is susceptible to noise present on raw unprocessed image data. First, the raw image is convolved with a Gaussian filter. The result is a slightly blurred version of the original which is ideally not affected by a single noisy pixel to any significant degree. Secondly, an edge in an image may point in a variety of directions, so the Canny algorithm uses four filters to detect horizontal, vertical and diagonal edges in the blurred image. The edge detection operator (Roberts, Prewitt, Sobel for example) returns a value for the first derivative in the horizontal direction $\left(G_{y}\right)$ and the vertical direction $\left(G_{x}\right)$, as shown in (4) and (5).

$$
\begin{aligned}
& G=\operatorname{sqrt}\left(G_{x}^{2}+G_{y}^{2}\right) \\
& \Theta=\arctan \left(G_{x} / G_{y}\right)
\end{aligned}
$$

The edge direction angle is rounded to one of four angles representing vertical, horizontal and the two diagonals $(0,45,90$ and 135 degrees for example). Given estimates of the image gradients, a search is then carried out to determine if the gradient magnitude assumes a local maximum in the gradient direction. So, for example, 
- if the rounded angle is zero degrees the point will be considered to be on the edge if its intensity is greater than the intensities in the north and south directions,

- if the rounded angle is 90 degrees the point will be considered to be on the edge if its intensity is greater than the intensities in the west and east directions,

- if the rounded angle is 135 degrees the point will be considered to be on the edge if its intensity is greater than the intensities in the north east and south west directions,

- if the rounded angle is 45 degrees the point will be considered to be on the edge if its intensity is greater than the intensities in the north west and south east directions. This stage referred to as non-maximum suppression, produces a set of edge points in the form of a binary image.

Thresholding with hysteresis requires two thresholds - high and low. Making the assumption that important edges should be along continuous curves in the image allows us to follow a faint section of a given line and to discard a few noisy pixels that do not constitute a line but have produced large gradients. Therefore we begin by applying a high threshold. This marks out the edges we can be fairly sure are genuine. Starting from these, using the directional information derived earlier, edges can be traced through the image. While tracing an edge, we apply the lower threshold, allowing us to trace faint sections of edges as long as we find a starting point. Once this process is complete we have a binary image where each pixel is marked as either an edge pixel or a non-edge pixel. The edge detection algorithm is used as it is time efficient to implement and though personal experience has proven to revel distinctive features from the geo-located frames that will be selected later in section 3.1.4.

\subsubsection{Map Image Selection}

Using specific criteria, frames are selected from the video that will be used as the geolocated frames in the localization part of the solution. Such criteria include:

- Maximizing detail of a structure (e.g. houses)

- Minimizing trees

- Minimizing dynamic elements (e.g. cars, people, etc.)

The coordinates are then found on the map that correlate to the aforementioned selected frames, then they were label on the map to complete its construction. 


\subsection{Localization}

After the map has been created, one can localize the position of the vehicle by processing the video data that is recorded as the vehicle moves along the path. For every image frame taken at time step $t$ from an on board camera, the correlation function is calculated:

$$
k_{i}(t)=I C\left(I_{t}, I_{m i}\right) \text { for } i=1 \ldots n
$$

Where $k_{i}(t)$ the correlation between image $I_{t}$, at the current frame at time step $t$ provided by the vehicle camera, and image $I_{m i}$ the ith geo-located selected image frame from the map, $n$ represents the number of geo-located points in the map. Once all of these values have been calculated the values can be stored such that:

$$
K(t)=\left[k_{1}(t), k_{2}(t), k_{3}(t), \ldots, k_{n}(t)\right]
$$

$K(t)$ is then used in the map image match determination to create $H(t)$ as defined such that:

$$
H(t)=\left[h_{1}(t), h_{2}(t), h_{3}(t), \ldots, h_{n}(t)\right], \quad h_{i}(t) \in[0,1]
$$

Where $h_{x}(t)$, represents if a match has been determined with the $x^{\text {th }}$ geo-located, at time $t . H(t)$ is needed for the particle filter to localize the position of the vehicle. The following flow chart explains the necessary steps that need to be taken to accomplishing the localization portion of the solution. 
- Capture video $V_{L}$ while driving vehicle along path

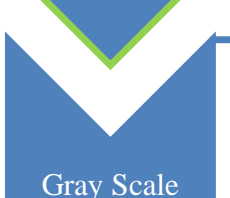

- Convert $V_{L}$ to Gray Scale

Gray Scale

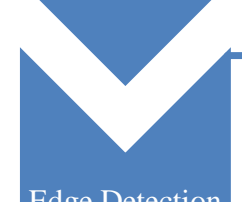

- Run Canny Edge Detector over $V_{L}$

Edge Detection

- Run Image Correlation on $V_{L}$ using geo-located frame from map as a base image

Correlation

- Repeate over all geo-located frames from map $M$ to generate $K(t)$

- Use $K(t)$ to generate $H(t)$

Determination

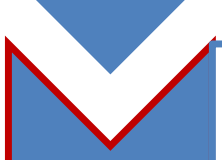

Particle Filter

- Use $H(t)$ to correct particle positions in a one-dimential particle filter to determine poosition estimates $\left[x_{\text {est }}(t), y_{\text {est }}(t)\right]$

\section{Figure 4: Localization block diagram}

The above block diagram demonstrates how the localization process is split into six phases;

Capture, Grey Scale, Edge Detection, Correlation, Determination, and Particle Filter.

\subsubsection{Video Capture}

Images must be captured as the vehicle is driven down a specific length of road. Similar to configuration as in section 3.1.1 


\subsubsection{Grey Scale}

Using computer vision and/or video processing tools, the video gets converted to grey scale. See details from section 3.1.2.

\subsubsection{Edge Detection}

Using computer vision and/or video processing tools, a Canny edge detection filter is applied over all of the frames of the video. See details from section 3.1.3.

\subsubsection{Correlation}

A combination of the image correlation functions provided in the Image Correlation and Tracking Library [20], were used to calculate the strain of features within the frames over time. The correlation process uses discretized grid sections to measure how similar or different two images are. Once an image has been discretized each grid cell is tracked for properties that would constitute a match. The properties are the number of line sections and relative position of features. The differences to an assumed fit(s) of the aforementioned properties are measured as strain along a single direction (x-axis). The movement of the grid sections is averaged and a line is fitted to the different positions. When the slope of this fitted line is close to zero it reflects an image frame in which there is minimal displacement (or differences) between the base image and the image being compared. The result is the average strain slope over each one of the frame pairs being correlated. This data, $K(t)=K(t)=\left[k_{1}(t), \ldots, k_{n}(t)\right]$, is used by the next phase (section $3.2 .5)$.

\subsubsection{Map Image Match Determination}

After obtaining strain $K(t)$ from the image correlation process, it must be processed to determine matches $H(t)$ between current frames and map frames. This data is then used as input data for the particle filter explained in section 3.2.6. For each map image strain calculated, there 
is random fluctuation due to the fact that the geo-located images, and the frames from the localization data set, are not identical. The data is smoothed and the derivative taken. There exists plateaus in the strain time plots and the goal is to try and find these plateaus as part of the data image match determination. Each one of these plateaus occurs at (or around) where there is a match in the image correlation process. This occurs because the change in strain is minimal when the base image is correlating well with specific frames in the localization data set. The derivative of the data will reveal sections of the data where the slope is close to zero, which represents a match in the image correlation. These steps are summarized below:

- Smooth out variance

- Derivation

- Smoot Derivative

- Find regions within threshold of zero

\subsubsection{Particle Filter}

As a visual demonstration of the localization process, a one dimensional particle filter is constructed to take the matching results, $H(t)$, in as input and show a visual representation of the vehicle moving though time (frames) and depict its actual and estimated position on a graph. A particle filter estimates an agent's position using the following pseudo code:

\section{Particle Filter Pseudo Code}

FOR $t=1$ to NumberOfFrames

Propagate Particles

IF MATCH

FOR $i=1$ to NumberOfParticles

Update weights according to Equation 4

END FOR

FOR $i=1$ to NumberOfParticles

Normalize weights using Equation 5

Add particles $\mathrm{x}$ times to temp array, where $\mathrm{x}$ is higher depending on weight of particles

END FOR

FOR $i=1$ to NumberOfParticles

Resample by randomly selecting particles from temp array

END FOR 


\section{END IF END FOR}

When a match in the data is found, the weights of the particles are calculated and the particles are re-sampled. Over each frame, the particles are propagated using a motion model which adds randomness in its movement. The weights for the particles in the particle filter are calculated using (10) and the standard deviation, $\sigma_{i}$, of matches for a geo-located image, where the match occurs, and the distance from where a match is discovered and where its actual frame number is.

$$
\begin{gathered}
\sigma_{i}=\operatorname{stddev}\left(h_{1 i}, h_{2 i}, \ldots, h_{j i}\right) \quad \begin{array}{l}
\text { for } i=1 \ldots n \\
j=1 \ldots \text { numOfMatches in } V_{m}
\end{array} \\
D_{i}(t)=X(t)-\operatorname{Goal} X(i) \\
W(p)=\frac{1}{\sqrt{2 \pi} \sigma} e^{\left(\frac{-D(t)^{2}}{\sigma^{2}}\right)}
\end{gathered}
$$

In which $h_{i}$ represents a match from the $i^{\text {th }}$ geo-located point and $n$ is number of matches for that geo-located point. The $t$ is the frame number where particle $p$ is at, and $W$ represents the weight of particle $p$. After all of the weights have been calculated all of the weights get normalized using the following function:

$$
W(p)=\frac{W(p)-\min W e i g h t}{\max W e i g h t-\min W e i g h t}
$$

The minWeight and maxWeight are found in the initial loop of calculating the weights. 


\section{Chapter 4}

\section{IMPLEMENTATION}

This section of the thesis explains how map creation and localization were implemented in an experiment. Titles of software, images, and other low level information will be used to explicitly explain how each of the phases of the experiment was accomplished. Several videos were recorded and two were selected to be used for the map creation and localization.

\subsection{Implementation of Map Creation}

The block diagram shown in Figure 2 demonstrates how the map creation process is split into five phases; Capture, Edit, Pre-Process, Extract, and Select. The following sub-sections describe how each one of the phases from the corresponding section in the solution was implemented in this experiment.

\subsubsection{Video Capture}

A Logitech Quick Cam Pro 5000 was used for obtaining all video footage. At the time of purchase, it had very good reviews and was one of the best web cameras for capturing fast motion without blurring images [21]. The Camera was attached using double sided tape to the passenger side window of a 1997 Honda CR-V (Figure 5). 


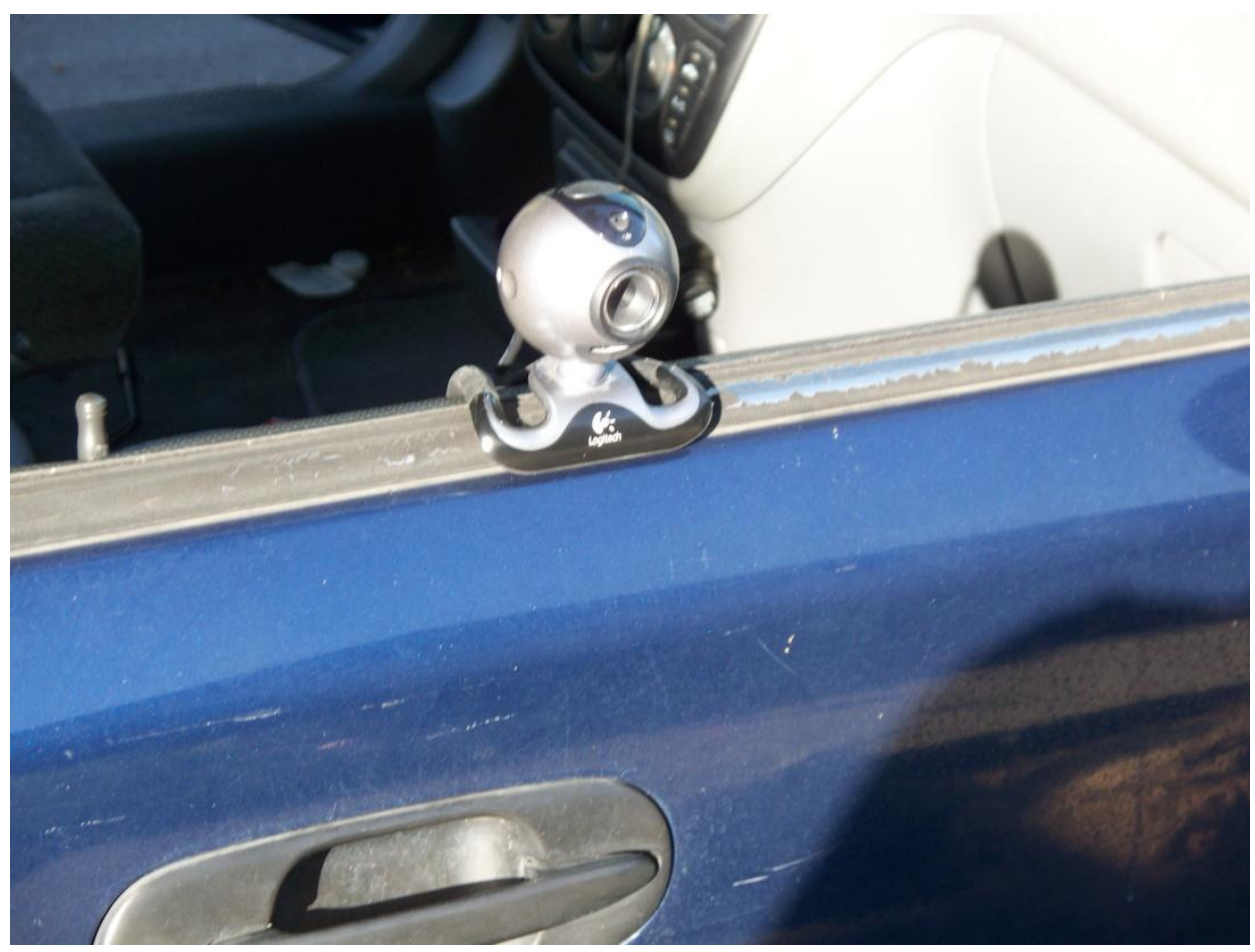

Figure 5: Camera Mounted

The selection of road selected for this experiment began just outside of 2600 El Cerrito Street, San Luis Obispo, California. The video was set to record and the vehicle was driven as slowly as possible (approximately 5 miles per hour) down El Cerrito Street, until the intersection of El Cerrito Street and El Paseo Street (as shown in figure 6) was reached. 


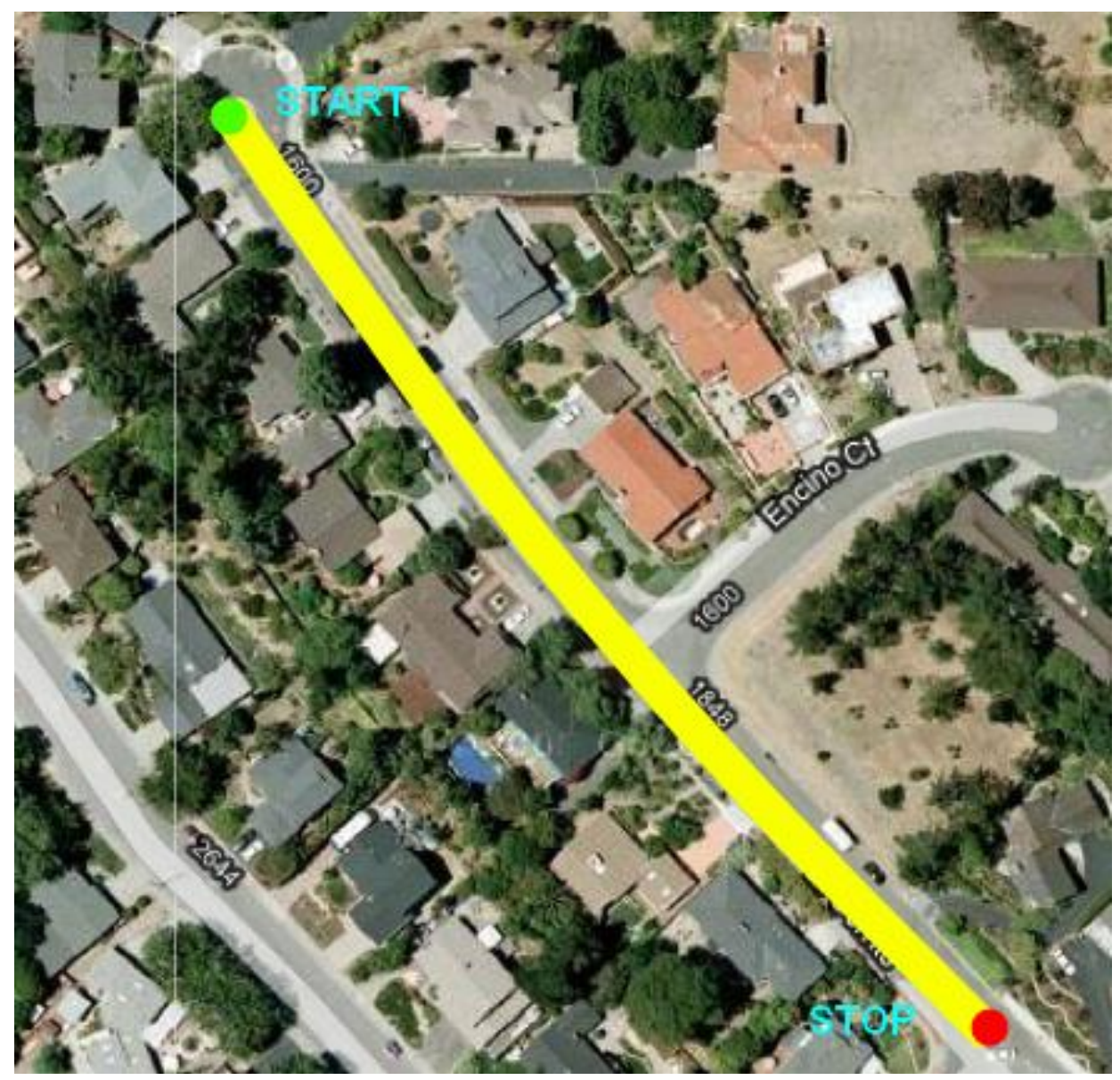

Figure 6: Route Map

\subsubsection{Video Editing}

Initially the video was opened and edited using Microsoft Windows Movie Maker from Windows Vista (not XP or Windows 7). This specific version was used because of its unusual stability while processing visual effects and for its ability to use both included and custom filters. These filters can seamlessly be applied to videos, and proved helpful in section 4.1.3. Using the trim function in Movie Maker (shown in figure 7), the video was trimmed to only include the visual data from the desired region of the experiment. 


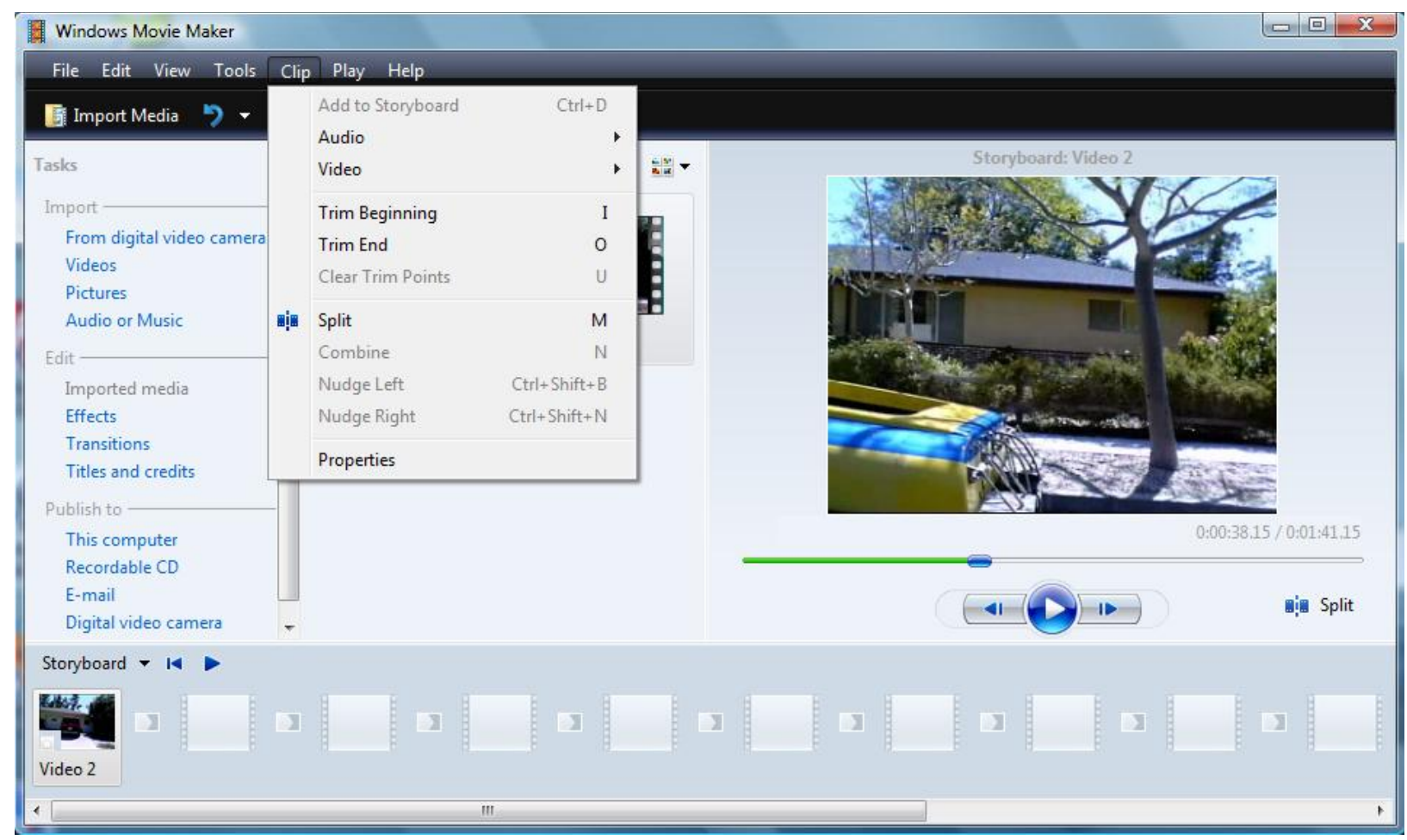

Figure 7: Trim Tool

\subsubsection{Grey Scale \& Edge Detection}

Using Microsoft Windows Movie Maker, the video was converted into grey scale (Figure 8). A Microsoft provided implementation of Canny Edge Detection filter was easily added to the Movie Maker program as a free add-on. This filter was then available as a selection choice from the visual effect menu (Figure 9). Applying the edge detection filter took several seconds to apply over an entire video, and had similar if not greater resolution and quality than the OpenCV alternative. 


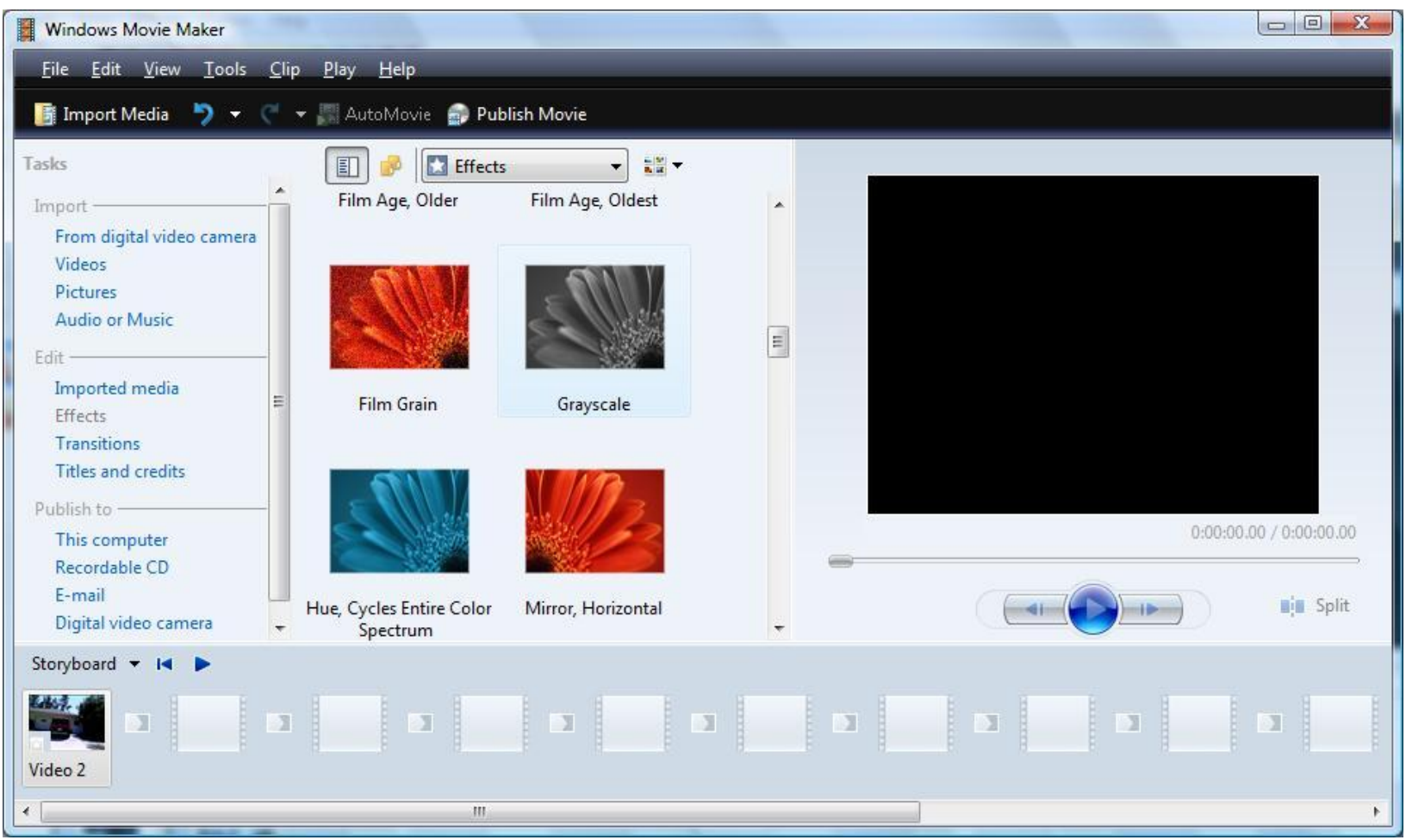

Figure 8: Grey scale Effect

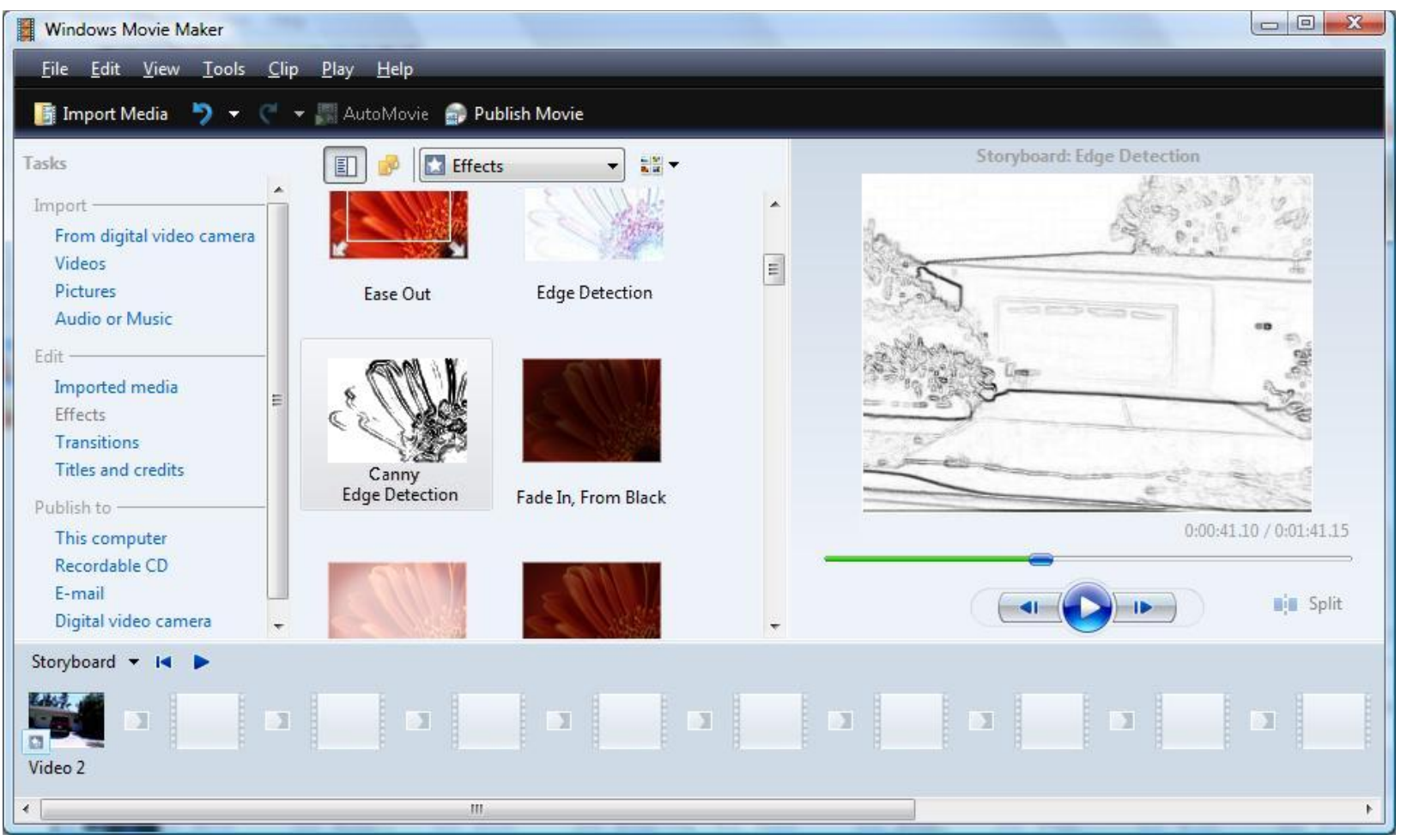

Figure 9: Canny Edge Detection 


\subsubsection{Convert Video Format}

In order to continue with the rest of the experiment, the video had to be converted from its original video format used in Windows Movie Maker (WMV) into a format in which no compression is used. This was done so that all of the frames could be extracted in section 4.1.5. This phase was not originally part of the solution but was a minor step in the implementation of the experiment. To do this, a program called WinAVI was used. WinAVI is designed to convert various video formats into DVD format, and vice versa. In this case the software was used to convert the WMV file into MPEG-2 video format (Figure 10). MPEG-2 format uses no video compression and thus allows the extraction of all the frames from entirety of the video.

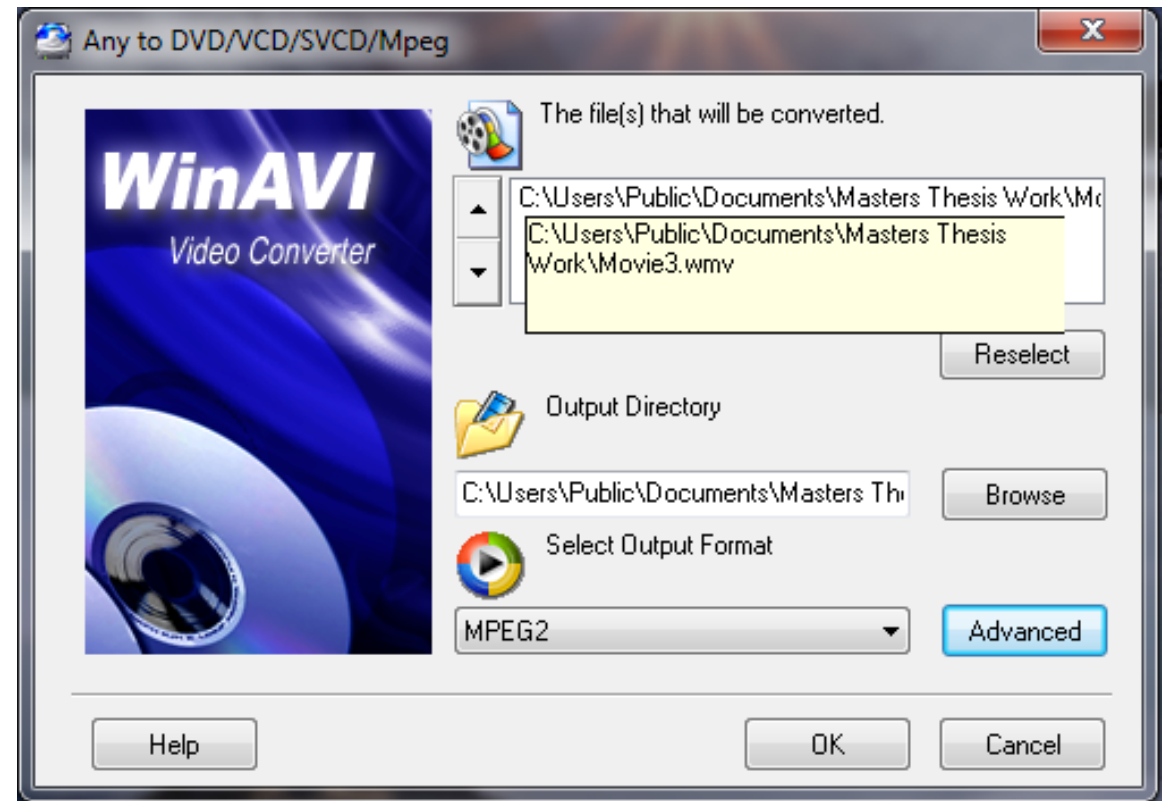

Figure 10: WinAVI Convert WMV to MPEG-2

\subsubsection{Image Extraction}

IrfanView, a multi faceted image application with batch conversion abilities, was used to extract all of the frames from the MPEG video file produced in section 4.1.4 (figure 11). This process apparently is not always without error. The program had failed multiple times in completing the extraction process. Such failures included skipped frame numbers, corrupted frames, or frames left blank. The solution to this problem was to repeat the process on multiple computers until the process was completed. The cause of this malfunction is yet unknown, but irrelevant to the true purpose of this experiment. 


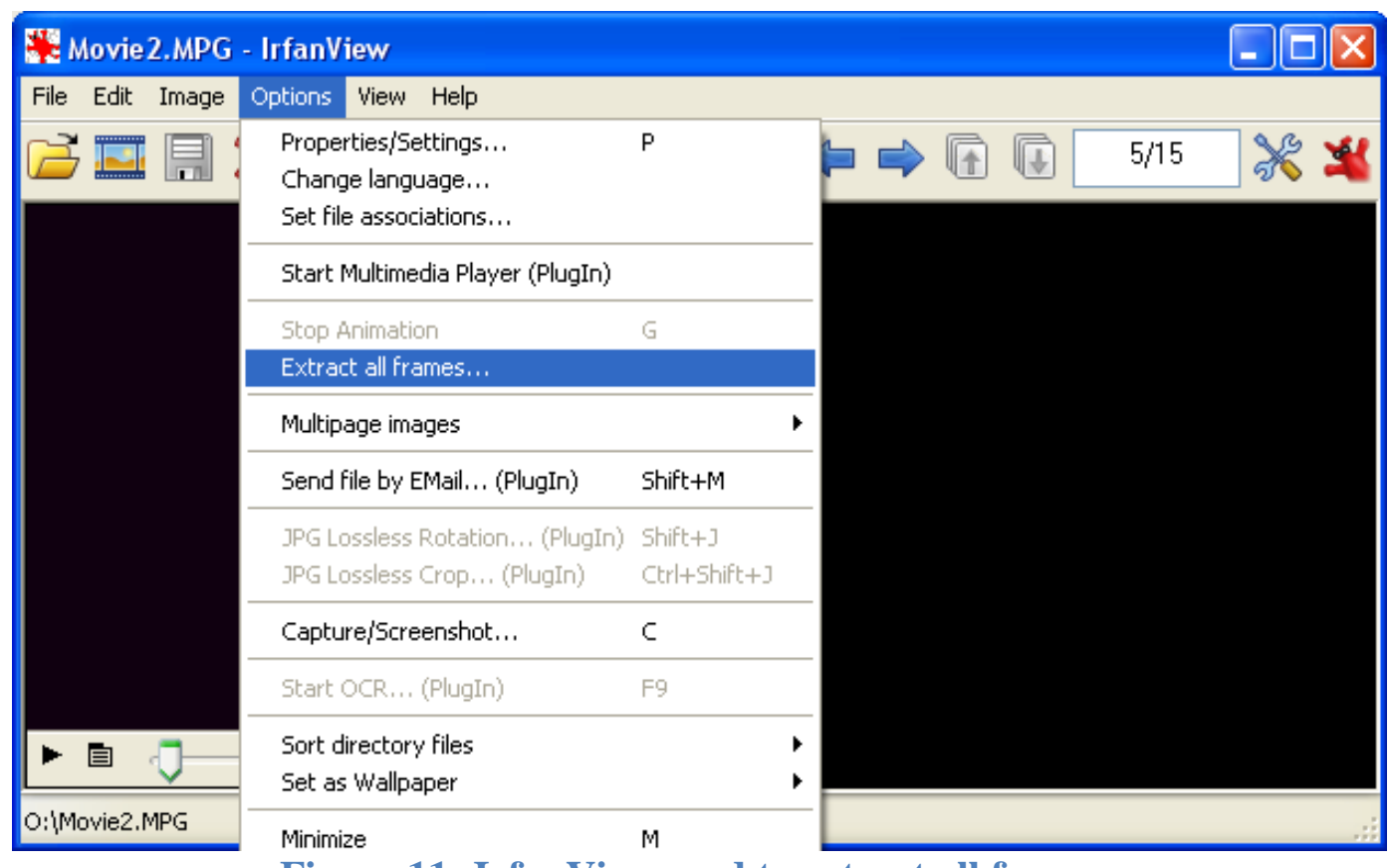

Figure 11: IrfanView used to extract all frames

\subsubsection{Map Image Selection}

For this experiment, five specific frames were selected from the video data set and their correlated geo-located points on the map were determined. These images were selected using the criteria discussed in section 3.1.4. These frames were labeled image 1 though 5 and are noted below by the image number as well as the corresponding frame number (figure 12). The geolocation information for each point was determined by matching the image with the view on Google Street View and finding the coordinates of that particular location, demonstrated in figure 13 and 14. 


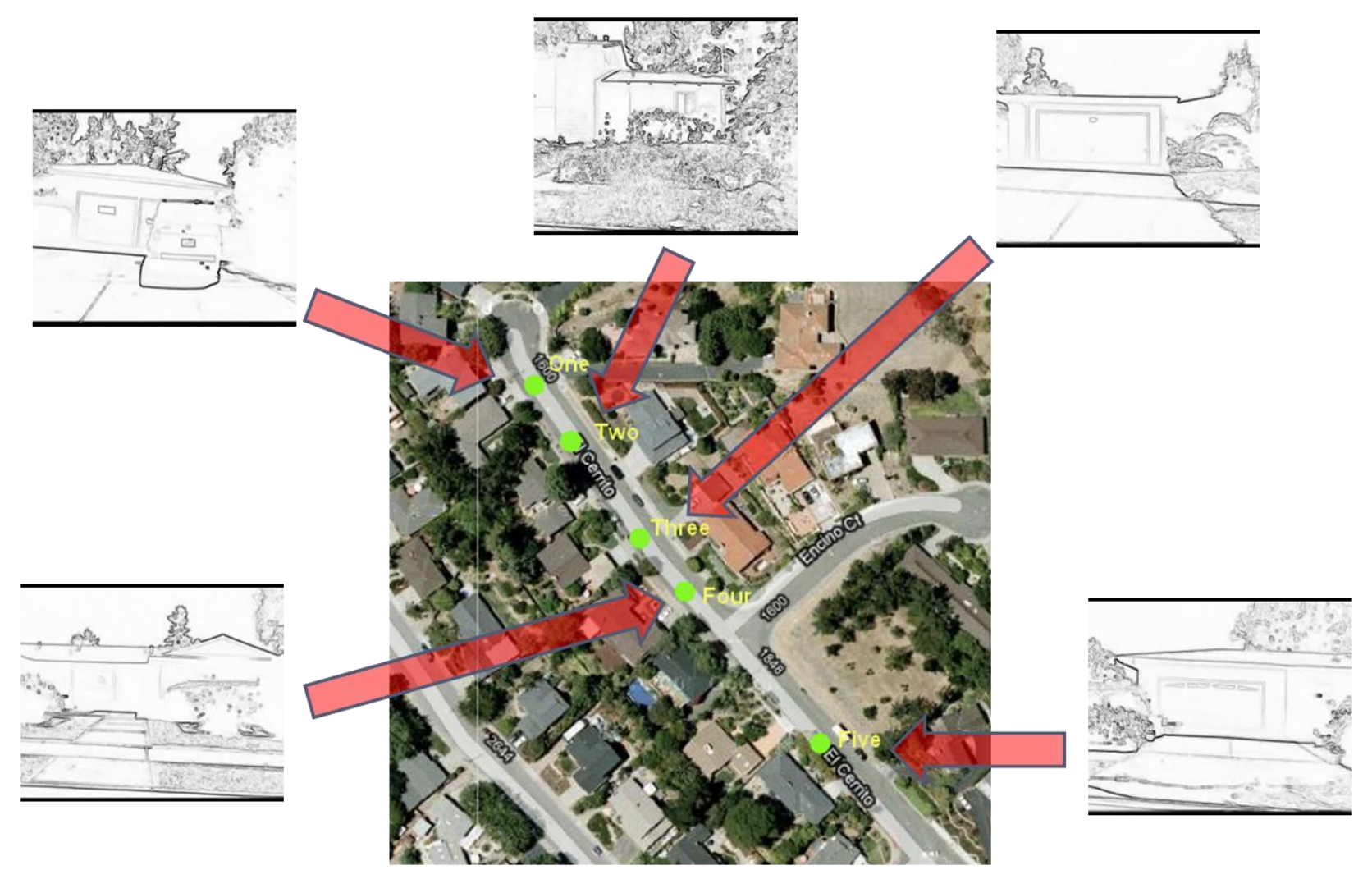

Figure 12: Geo-Located Image Locations

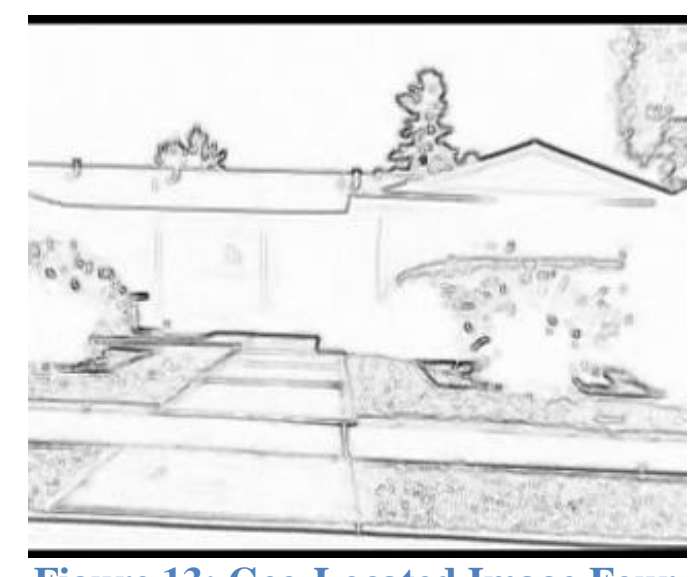

Figure 13: Geo-Located Image Four

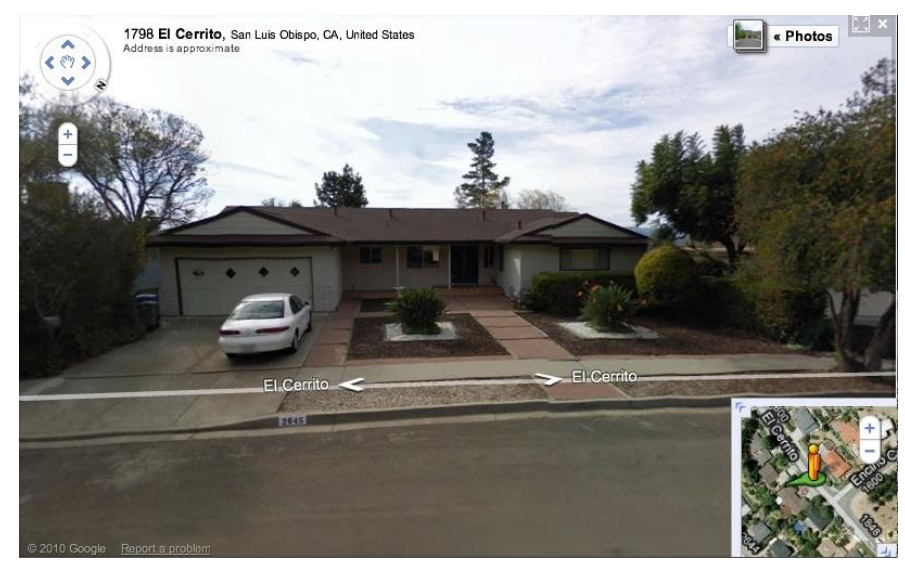

Figure 14: Google Street View Map Image 4

\subsection{Implementation of Localization}

The block diagram shown in Figure 4 demonstrates how the localization process is split into eight phases: Capture, Edit, Pre-Process, Extract, Correlate, Data, Graph, and Particle Filter. The following sub-sections describe how each corresponding phase of the solution was implemented in the experiment.. 


\subsubsection{Video Capture}

The same camera used in the map creation part of the implementation was once again attached using double sided tape to the passenger side window of a 1997 Honda CR-V (see Figure 3). Once again, starting just outside of $2600 \mathrm{El}$ Cerrito Street, the video was set to record and the vehicle was driven down El Cerrito Street (Figure 16) until the intersection of El Cerrito Street and El Paseo Street (Figure 4) had been reached.

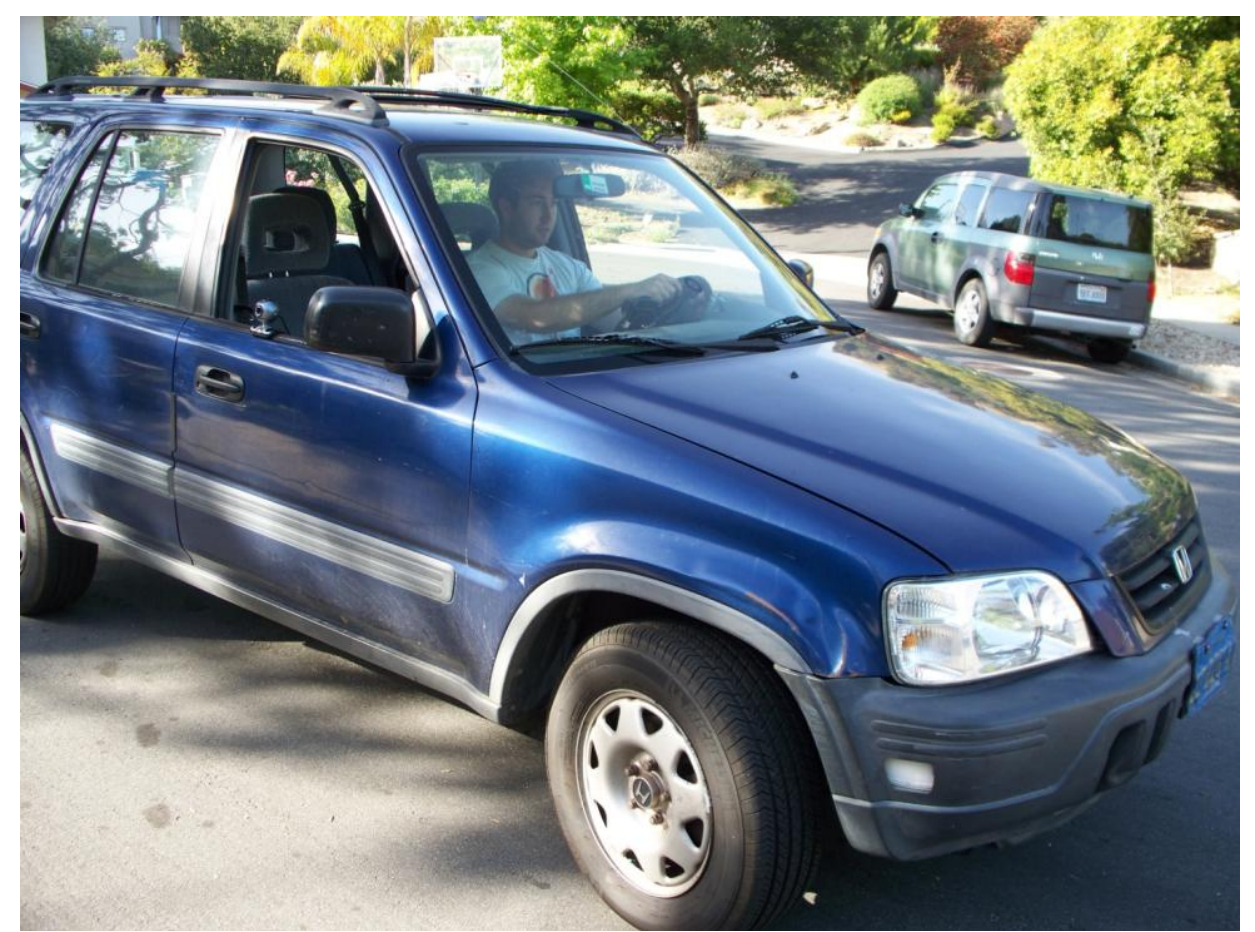

Figure 15: Start of Driving Run

\subsubsection{Video Editing}

The video was opened and edited using Microsoft Windows Movie Maker. Using the trim function in Movie Maker (shown in figure 5), the video was trimmed to include the visual data from the desired region of the experiment which corresponds to the desired region used in the map creation from section 4.1.2. 


\subsubsection{Grey Scale \& Edge Detection}

While still using Microsoft Windows Movie Maker, the video was converted into grey scale and had the Canny Edge Detection filter mentioned in section 4.1.3 applied. Applying the edge detection filter took a matter of seconds to apply over the entire video. Refer to section 4.1.3 for further detail on this process.

\subsubsection{Converting Video Format}

The WMV video file needed to be converted to MPEG-2. IrfanView was used to convert the WMV videos into MPEG-2 video format (Figure 10). For further information on this process refer to section 4.1.4.

\subsubsection{Image Extraction}

Similar to the step taken in section 4.2.5, IrfanView was used to extract all of the frames from the video data set. For more information on this process refer to section 4.1.5. This section differs from section 4.1 .5 because right after extracting the images from the video, the frame numbers that correspond to the geo-located frames from the map video had to be found. This is due to the fact that the exact speed throughout both of the videos cannot be regulated nor synced with one another. A visual inspection id used, the frames in the localization video are compared against the geo-located images from the map (Figure 16). When a match was found the frame number was noted elsewhere for use in the Particle Filter described in section 4.2.8.

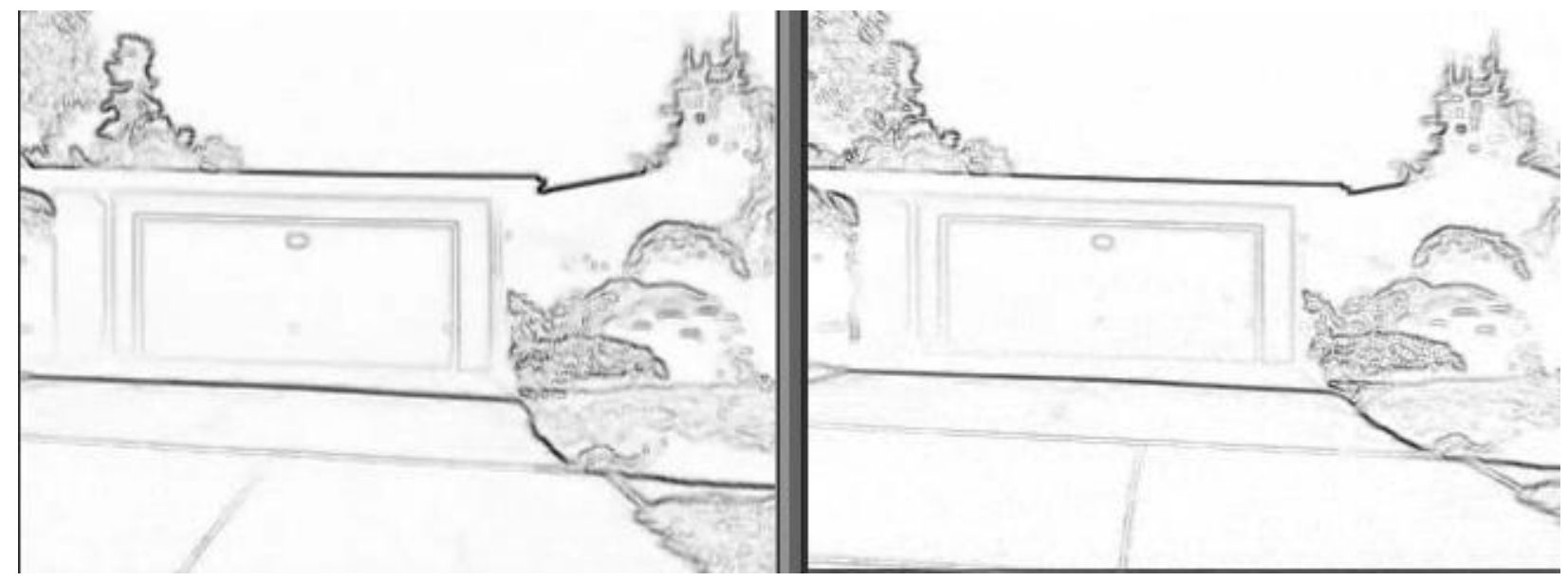

Figure 16: Visual Compare, 3rd Geo-located frame on left, Frame 587 from Localization Video on right 


\subsubsection{Image Correlation}

This particular phase implements the core image processing function of this solution. The process of running the localization video against a geo-located image from the map is actually a process that needs to be done five times; One time for each base image selected to be geo-located in the map creation. To accomplish all of the following tasks mentioned in this section, the Image Correlation and Tracking Library and functions must be installed into Matlab [20]. Initially, a "file list" has to be generated; This is a file that contains the names of all of the frame image files from the localization video. This is accomplished while in MATLAB by typing 'filelist_generator;' and pressing 'ENTER' at the command line of MATLAB. There are two ways to proceed now: Either choose to 'manually' type in the image numbers desired for processing or 'automatically' generate a list of images by pointing out the first image of the set, and the function will find all images with increasing number before the point in the name (e.g. 'PIC00001.Tif') (figure 17). The latter choice was selected and MATLAB was pointed to the first image of the set (dataset2_0001.tif). The function was then able to generate a list of the file names from “dataset2_0001.tif” all the way to "dataset2_1164.tif.

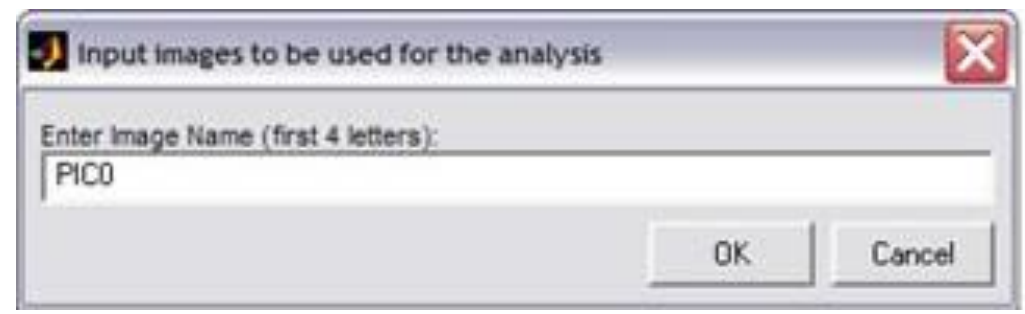

Figure 17: Pointing to the first image in a series

After the file list is generated, MATLAB asks where to save the list. In this case the files were saved to the same directory to which the library and images are found. (Figure 18) 


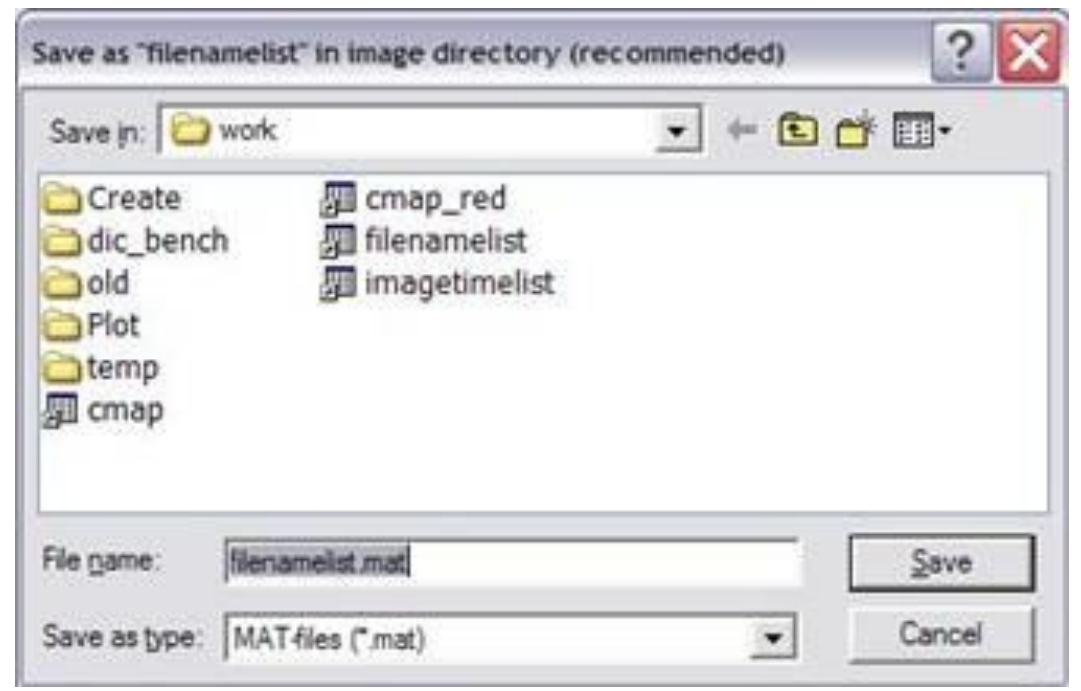

Figure 18: Saving file list

The next step in the image correlation process is to generate a grid (or section) of the frames that the correlation algorithms will be looking. It was decided early on in the experiment that the region of interest would be the top half of the frame as it included roof detail of the houses and avoided the region of the video that had shown cars either parked on the street or in the drive way. In the creation of the grid, one would be asked to point to the base image to which the images in the file list will be compared against. This is where the repeating steps would begin. Initially, the first geo-located image from the map would be selected which should correlate with the image from the localization data set at frame 88 and end with the last geolocated image from the map that correlates with frame 1052 from the localization data set. Once the image was selected a dialog box was presented that asks the user what type of grid they would like to make (Figure 19). 


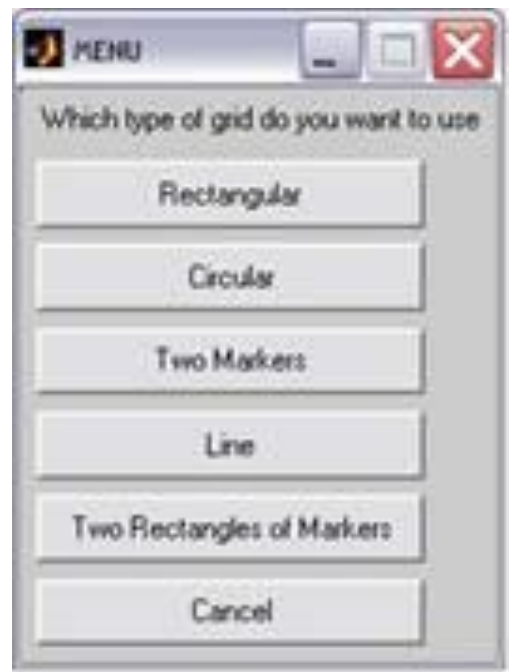

Figure 19: Select grid type

A Rectangular grid was selected for every image. After that selection was made an additional window appears that shows the base image that was selected. Using the mouse, the top half section of the frame was selected.

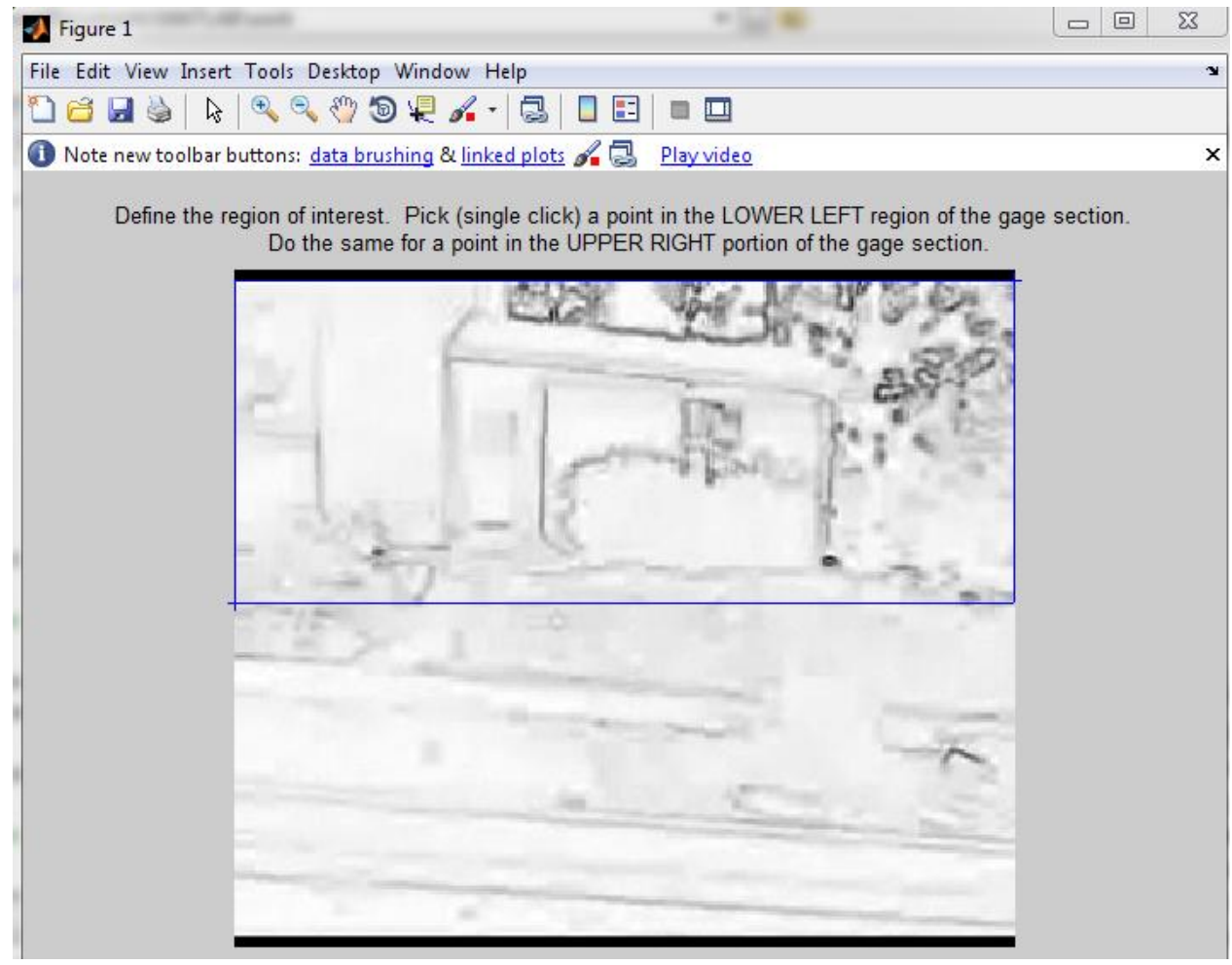

Figure 20: Grid Selection 


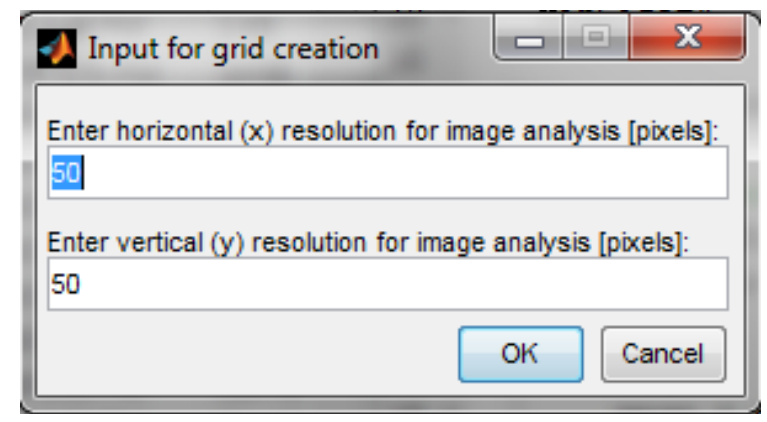

Figure 21: Grid Resolution

After selecting the region of interest, MATLAB then prompted the user for a desired $\mathrm{x}$ and $\mathrm{y}$ resolution (Figure 21). The default resolution is 50 by 50. Double the default resolution was used as the effective region of interest from the whole image had been cut down to half. Essentially, the section of the frame is being broken up into discretized parts that will be tracked and correlated later. After this was done, the grid files are created, and the image correlation function was ready to be executed.

The automation function is the central function and processes all markers and images. Therefore the 'Current directory' in MATLAB has to be the folder where automate_image.m finds the filenamelist.mat, grid_x.dat and grid_y.dat as well as the images specified in 'filenamelist.mat'. 'automate image;' is typed and the 'ENTER' key is pressed at the command line of MATLAB. At first, automate image opens the first image in the filenamelist.mat then 'automate_image.m' will process all images in the 'filenamelist.mat' and plot the grid with green and red crosses on top. The function is attempting to find similarities in the discretized sections made by the grid generation, from frame to frame, tracking their movement (or strain) over time (Figure 22). Depending on the number of images and markers you are tracking, this process can take between seconds and days. In this case it took about five minutes per base image to run the process over the entire localization data set. When all images are processed, automate image will write the files validx.mat, validy.mat, validx.txt and validy.txt. The text files are meant to store the result in a format which can be accessed by other programs and functions that can be used later and potentially in future applications. 


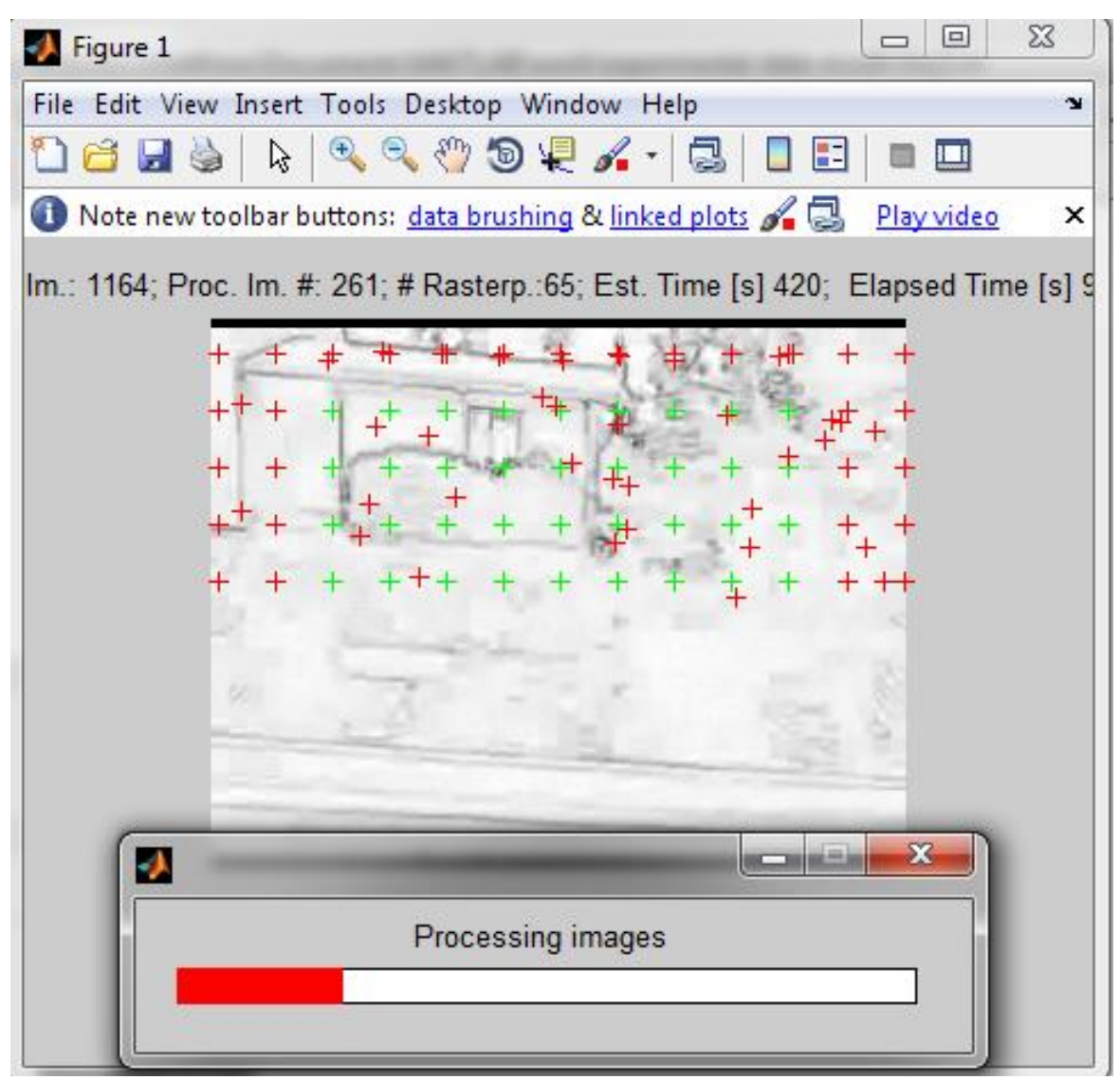

Figure 22: Automate Image Running

The final step involved with the image correlation and tracking library is to analyze the displacement and strain of the features calculated in the previous function. The post processing is the most interesting and rewarding step since one can analyze the collected displacement data. The displacement.m function is a small collection of functions which allows the user to review the displacement field, calculate the strain or delete markers which were not correlated or tracked very well. To start, one types "displacement" into the command window. A window will pop up asking for the validx.dat file which contains the x-displacement off all markers in all images, followed by a dialog for the validy.dat containing the y data. After "displacement.m" has loaded both files, a new window pops up that allows one to choose between different options of visualizing the data (Figure 23). 


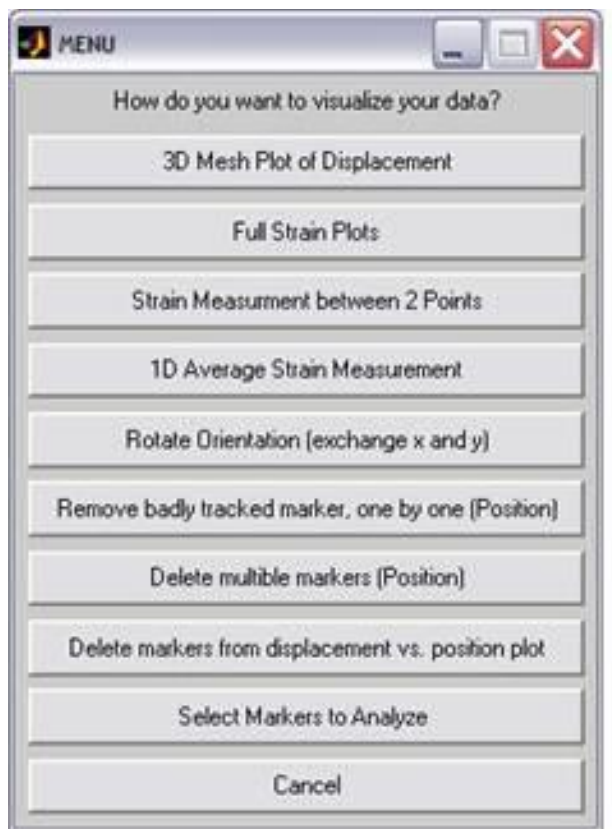

Figure 23: Visualize Data Menu

For the purposes of this experiment, the 1D average Strain Measurements are needed. After clicking on the "1D Average Strain Measurement" button, the x-displacement versus x-direction will be plotted for each image and then fitted by a linear function. The slope is the true strain which will be plotted versus the image number after all images are processed. If you choose to save the strain versus image number, you the data will be saved as an ASCII file which can be opened with MATLAB, Excel, or notepad. In this case the y-strain is negligible as the vehicle is moving predominantly horizontally through space.

The output of the final graph can then be saved as raw data. It is this data that gets smoothed and post-processed later is section 4.2.7 in order to demonstrate how this experiment can be a viable means of autonomous localization.

\subsubsection{Map Image Match Determination}

The data that is obtained from the previous section depicts a plateau that occurs in the plotting of strain for each of the base images. The goal is to find all of these plateaus as part of the map image match determaination method. One of these plateaus should occur on or around where there is a match in the image correlation process. This happens because the change in strain is minimal when the base image actually is correlating well with specific frames in the 
localization data set. There is an entire set of data for each one of the results from the previous section, containing small amounts of error, and expected random fluctuation due to the fact that the geo-located images, and the frames from the localization data set, are not identical. Steps from section 3.2.5 are used to smooth the data in question and to pick out the regions where these plateaus appear. [Appendix A] The code is designed to remove small amounts of fluctuation to make the graph smoother and to extract regions in which the slope of the graph is close to zero. The smoothing section is done by creating a small window and averaging the points within each window across the entire set of points. Once the graph has been sufficiently smoothed, MATLAB takes the derivative of the graph and locates points where the slope is close to 0 within another window. The desired final result is a set of data that has a domain of 1 to 1164 , representing the frame numbers, and a range of 0 or 1 . The 1's get placed in areas in which are considered a match for that image, and 0's elsewhere. For example, if the data from the first results data processing reads that image 1 has been found at frame 110 then a 1 would be the value at index 110 out of 1164 . In reality, the frame is taken at frame 118, so 110 would be quite close. This match determination method is run on each one of the resulting data sets retrieved from section 5.2.6 (a total of 5 times). The resulting data sets can be graphed one on top of another in different colors to show when the whole system thinks the vehicle is at a certain point at a certain time. In addition, this data is pipelined into a one-dimensional particle filter to visually demonstrate the viability of this system.

\subsubsection{Particle Filter}

Using the Post-Processed data from the previous section and the value of $\sigma_{\mathrm{i}}$ calculated by taking the standard deviation of the matches in $H(i)$ using Microsoft Excel, a one-dimensional particle filter (as described in section 3.2.6) was constructed in MATLAB [Appendix B]. Once this algorithm was implemented into MATLAB, it became a simple deed of pulling the data from section 4.2.7 into the Particle Filter. The particle filter visually shows over time (frames) the estimated position of the vehicle in $1 \mathrm{D}$ space as well as the error in position estimation over time. 


\section{Chapter 5}

\section{RESULTS}

The following are the results of the image correlation between map images one though five with each frame of a second video set, $\mathrm{V}_{\mathrm{L}}$ (Section 4.2.6).

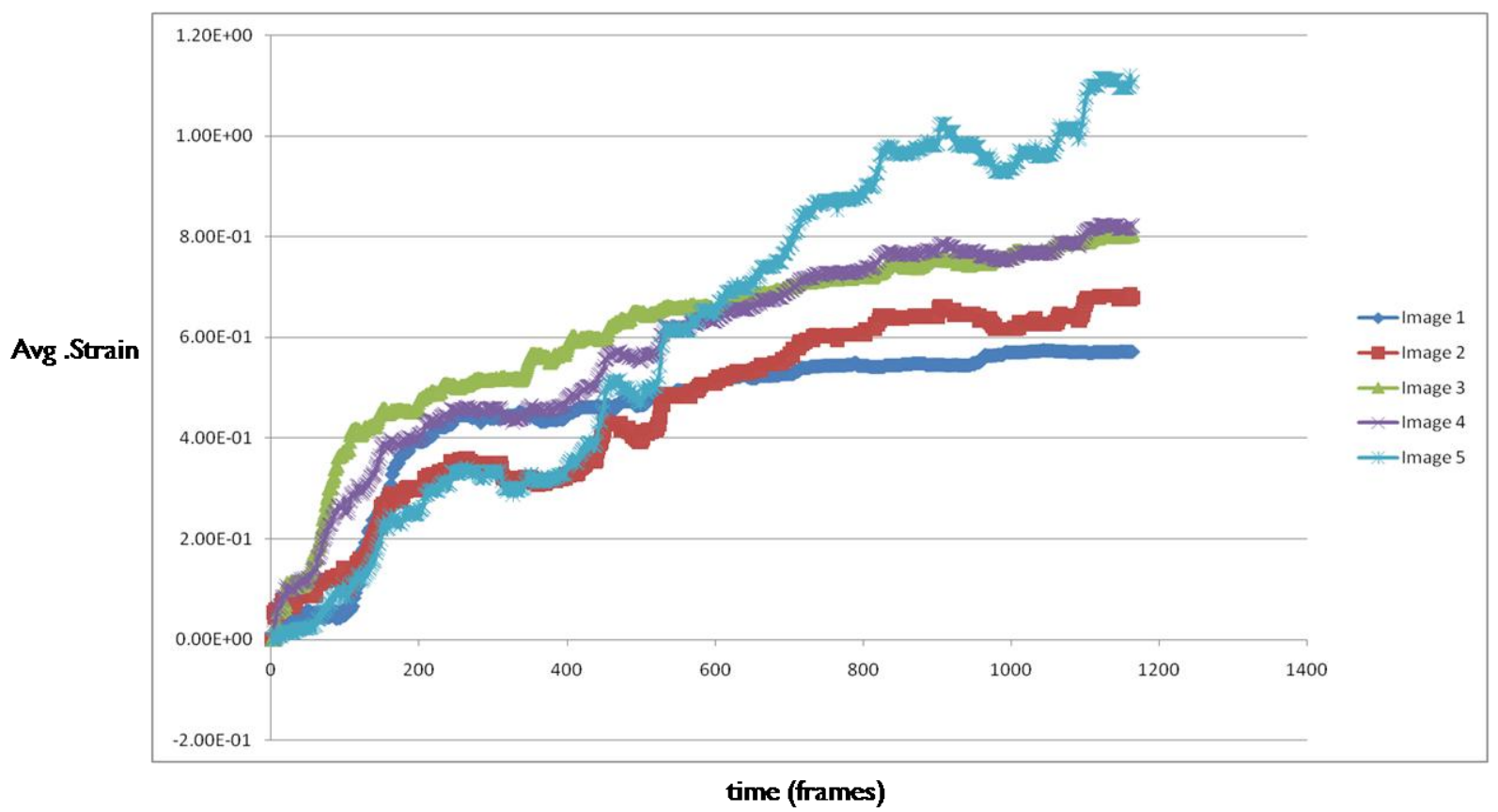

Figure 24: Image Correlation Strain

Figure 24 above represents strain (y-axis) over frames (x-axis). While some of the plateaus are easy to distinguish in the above figure (Figure 24), there is enough variance and random error to warrant the use of the data processing described in section 4.2.7. Image matching described in section 4.2.7 produced matches shown in figure 25 . 


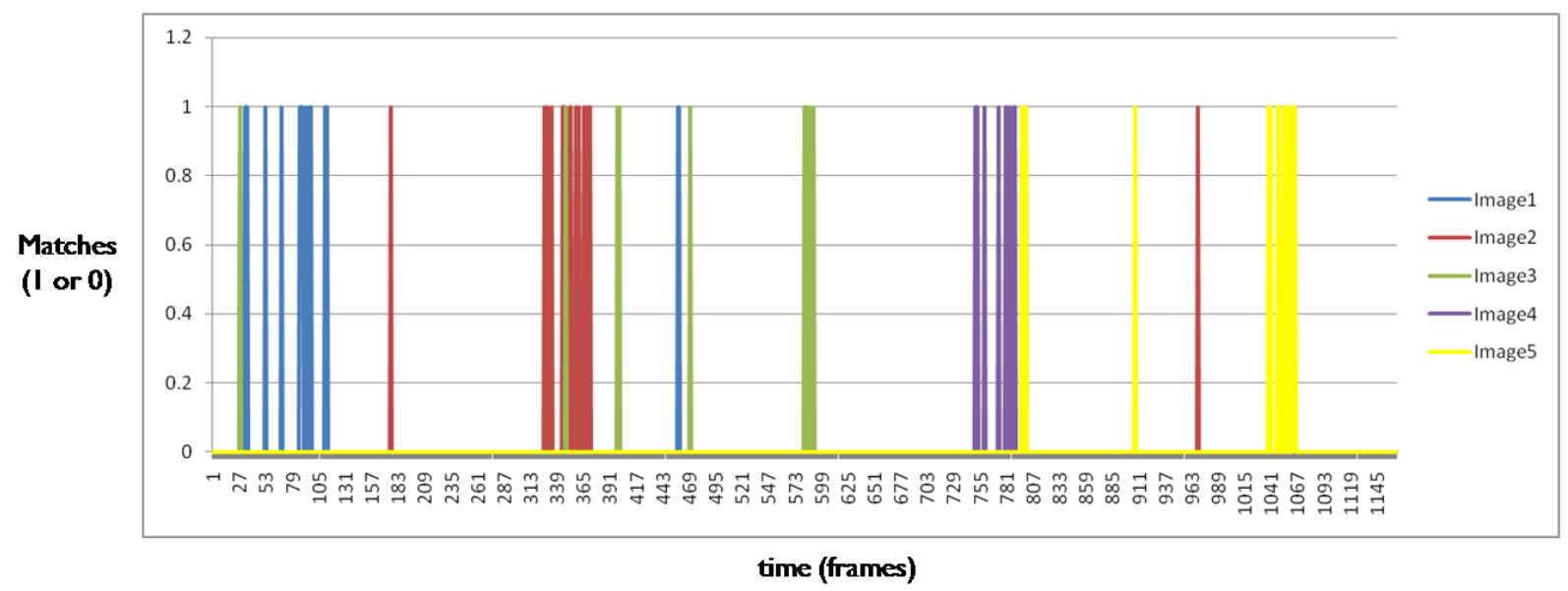

Figure 25: $K(t)$ results for each geo-located image

Figure 25 above shows matches as a function of time (frames) in the $\mathrm{x}$-axis. Figure 26 shows when a match is detected for each of the geo-located images over time (frames). It can be seen that there are a few false positives but the matches generally cluster are around the map image location:

- Image $1 \Rightarrow$ Frame 88

- Image 2 => Frame 353

- Image $3=>$ Frame 587

- $\quad$ Image 4 => Frame 777

- Image 5 => Frame 1052

These frame numbers were found using visual comparison with the map's geo-located images explained in more detail in section 4.2.5.

This data shows in a simple yes or no ( 1 and 0 respectively) form, if the system thinks that the vehicle is at the point of the indicated image at a specific time value (frame number). This data is further utilized as it is fed into the simple one-dimensional particle filter. The results of that filter are demonstrated by showing some select frames of the Particle Filter animation, displayed below (Figures 26 through 29), where the particles are represented by the red circles and the actual position is the blue line, the $y$-axis is irrelevant, and the $x$-axis is time (frames): 


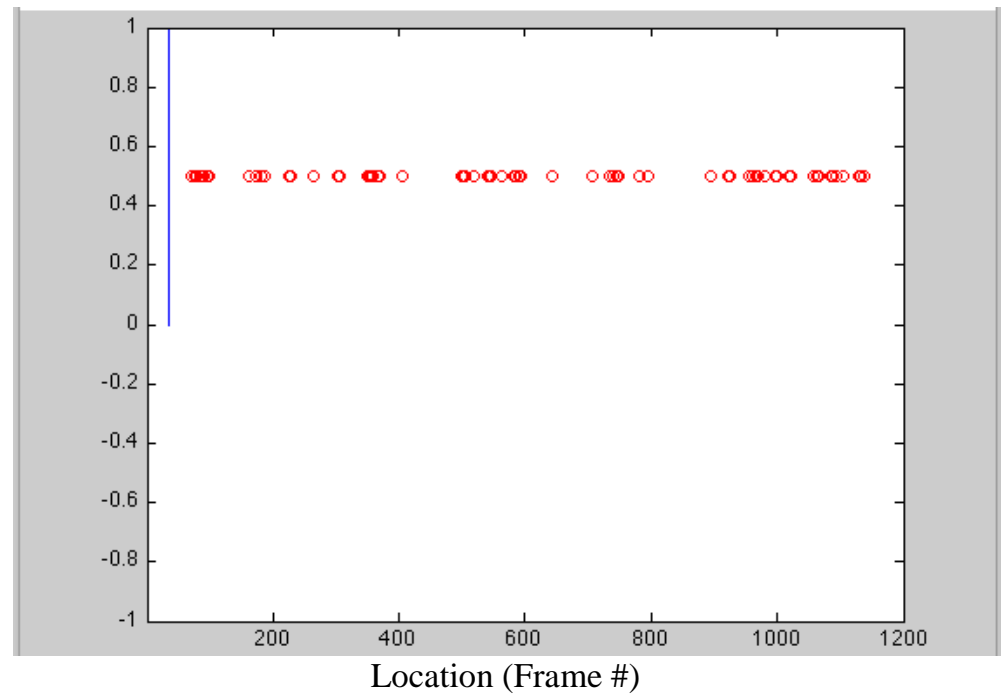

Figure 26: Initial State of Particle Filter

It can be seen in figure 26, near the very beginning of the localization the particles can be found randomly dispersed over the domain. This is because the particles the initial position is unknown. The actual location is indicated by the blue line and the particle locations are indicated by the red circles. The Results improve as the vehicle progress though time (frames).

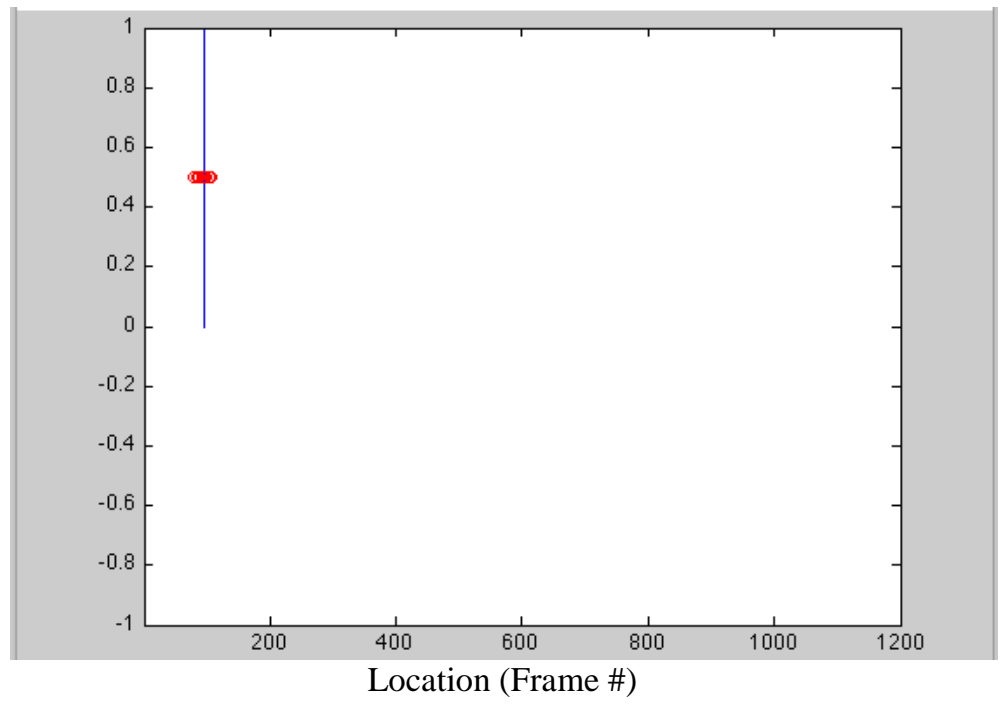

Figure 27: First Grouping at $t=95$

Figure 27 shows how the particles group around the actual position of the vehicle early on. 


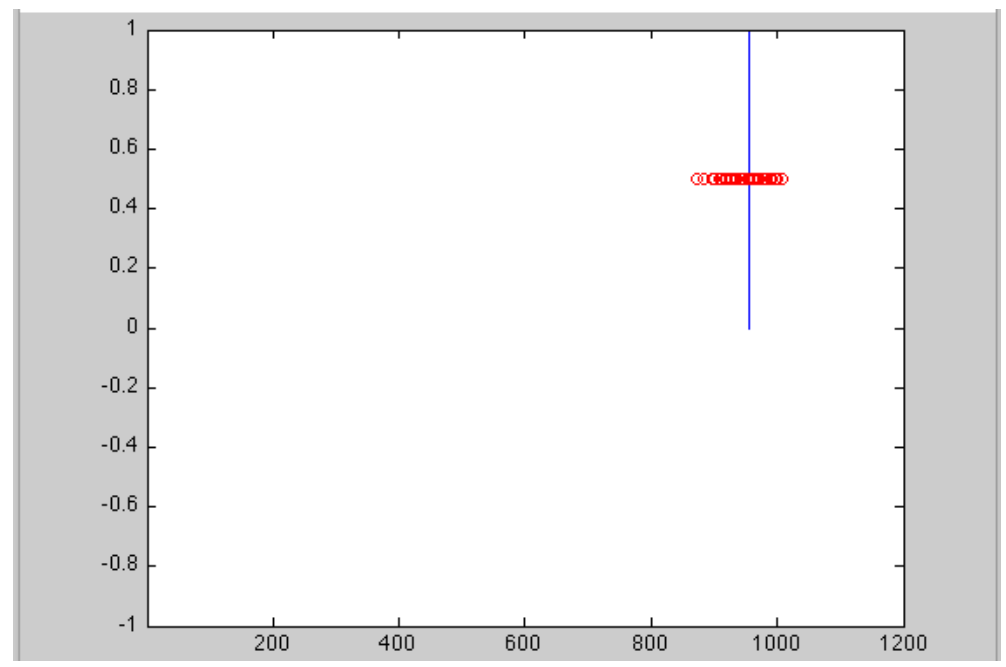

Location (Frame \#)

Figure 28: A bit of random dispersion at $t=955$

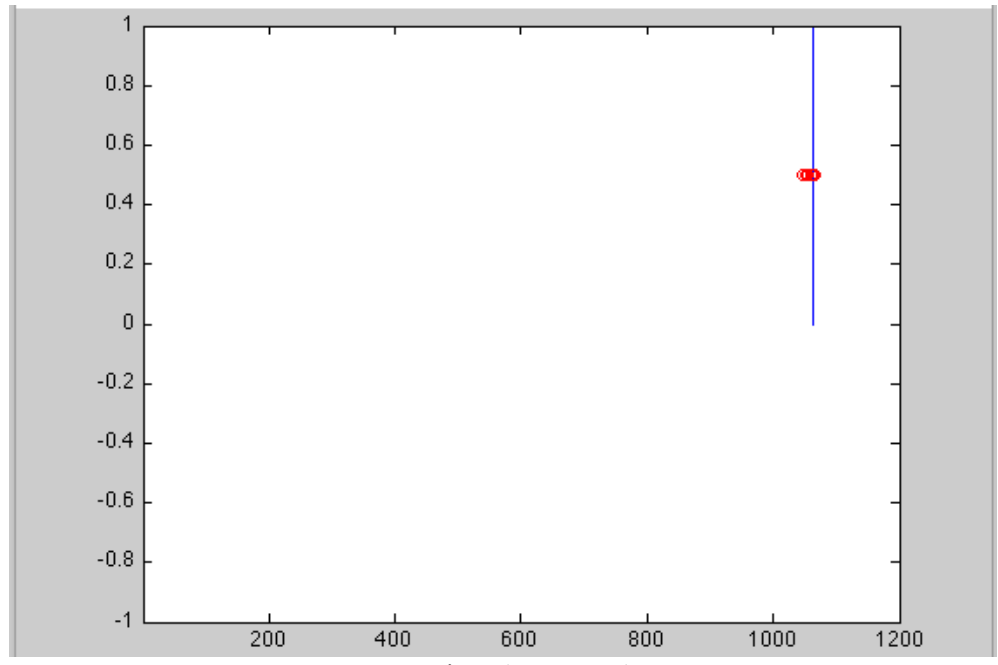

Location (Frame \#)

Figure 29: Particle Filter Right after a good set of matches at $t=1055$

Figure 28 shows a wide dispersion of particles that still average close to the blue line. This dispersion occurs cumulatively in regions where there are no matches in the data, but can be seen once again forming a tight grouping in figure 29 , as the particles get re-sampled due to the matches of the $5^{\text {th }}$ image. As the particle filter is running, the position of all the particles is averaged, and the error from that average (estimated position) compared to the actual position is stored. 


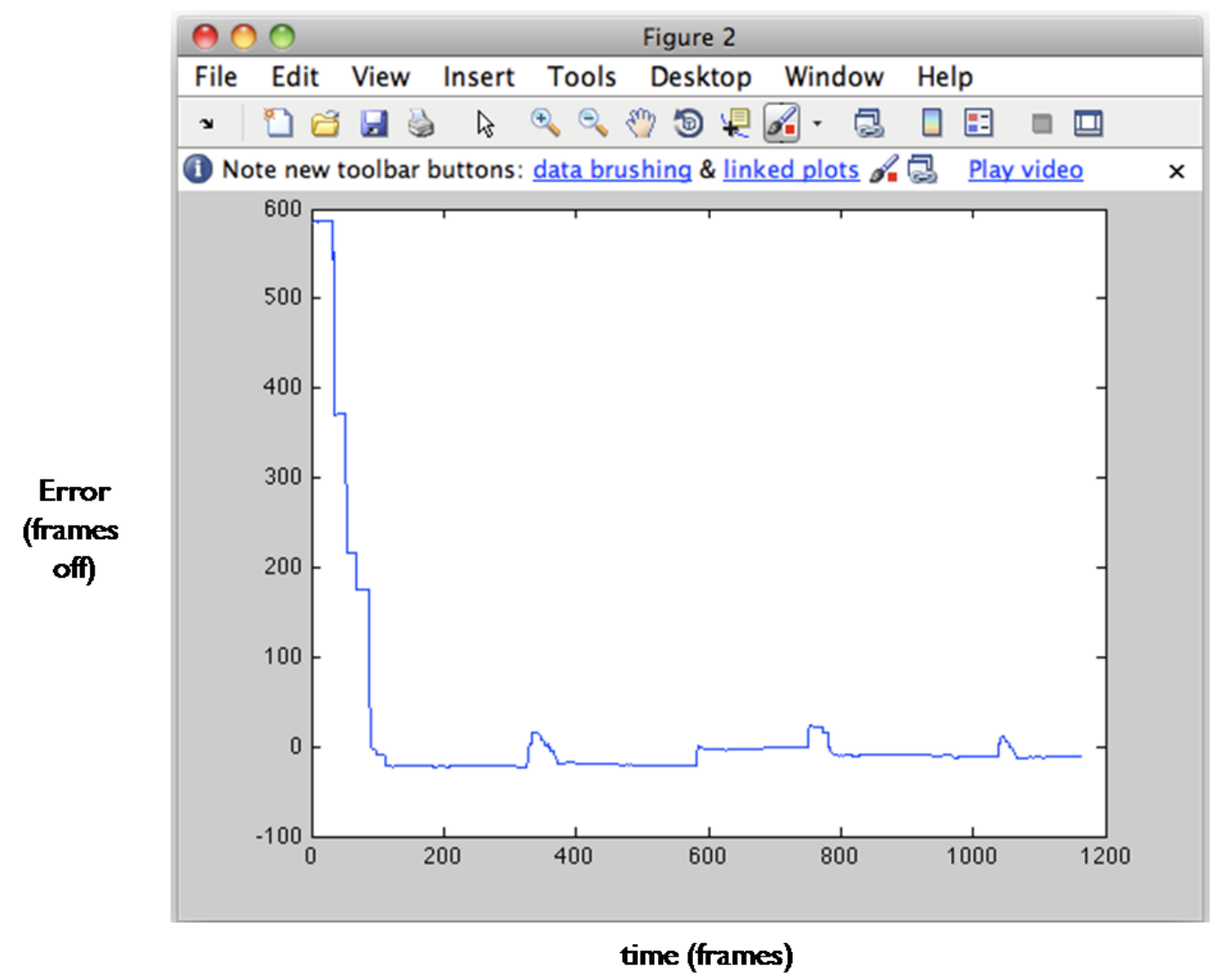

Figure 30: Error Graph

Figure 30, above, shows the error (y-axis) in number of frames off, over time (x-axis) in units of frames in the video. It can be seen that as soon as the localization data revels a good match, the error very quickly drops down to essentially zero, and continues to stay in that area over time. 


\section{Chapter 6}

\section{CONCLUSION}

It can be seen in the data shown in chapter 5 that the particular configuration of technology described in this thesis is a viable means of accomplishing autonomous localization. The image correlations technology successfully finds matches between any two images. The particle filter not only shows how one can localize a vehicle's position from a known starting point, but demonstrates how the particle filter can be implemented to satisfy the Kidnapped Robot scenario, where the initial position is unknown.

Experiments were a successful in that the position error decreases towards zero over time. The results demonstrate the ability to determine within $X_{m}$ where the vehicle was as a function of time while the car was in motion for this experiment. This experiment has shown the potential for this system to be scaled up and to potentially aid in addressing common issues with autonomous outdoor localization.

While not currently implemented in real time, it is expected that real time localization is possible if one or more changes are made. It is believed that current hardware couldn't process the data fast enough to perform the necessary calculations in real-time. With fast enough hardware, and perhaps changes to the correlation and other processing functions, there exists the possibility that certain parts of the implementation can be changed or perhaps upgraded in multiple ways to accomplish this future goal. Additional ideas for further improvements are contained within the next chapter of this thesis. 


\section{Chapter 7}

\section{FUTURE WORK \& POTENTIAL APPLICATIONS}

A relatively new feature of Google Maps is an interesting application called Google Street View. Google Maps and Google Earth have proved incredibly popular with Internet users and are used by public and private businesses and individuals as essential and informative tools. Street View provides an added experience by enabling users to see a location as they would if they were standing on the street. [8] There are huge benefits from the Street View feature, ranging from the promotion of tourism, to helping people find local businesses or meeting points, assisting property buyers and prospective tenants. [8] My assumption is that such a database like the one mentioned above which provides one with the street view, could be loaded dynamically as one is driving, and in the event that the GPS signals start degrading or fail, the software within the GPS unit, combined with a camera, could continue to localize one's position by comparing its images that it is recording with those of the Google Street View database.

There could potentially be an issue with constantly scanning large amounts of images so either smaller amounts of data would have to be sent at a time, or a faster storage medium could be used. The most likely method for satisfying this caveat is to use only the images located in the vicinity of GPS measurements. This is related to another issue; should the images and the respective location database be streamed via the Internet using a form of wireless broadband, or should a large storage medium be employed. Both ideas have their merits. Broadband seems more convenient and has the possibility of being updated as the database is updated. On the other hand, the storage medium queries would be faster, and would work in any area (ie. without the need for a wireless infrastructure). 


\section{Bibliography}

[1] S. Thrun, W. Burgard and D. Fox, "Probabilistic Robotics", MIT Press, 2005.

[2] R. Siegwart and I.R. Nourbakhsh, “Autonomous Mobile Robotics”, MIT Press, 2005.

[3] K. Y. K. Leung, C. M. Clark, and J. P. Huissoon, "Monocular Vision based Particle Filter Localization in Urban Environments", Proc. of the 2008 IEEE International Conference on Robotics and Automation, May 19-23, 2008

[4] W. Zhang and J. Kosecka, "Image based localization in urban environments," in Proceedings of the International Symposium on 3D Data Processing, Visualization, and Transmission, 2006.

[5] W. Wang, "Autonomous Control of a Differential Thrust Micro ROV”, University of Waterloo M.Sc. Thesis, 2006.

[6] S. Haykin. Kalman Filters, chapter 1, pages 1-21. John Wiley \& Sons, Inc., 2001.

[7] E. Royer, M. Lhuillier, M. Dhome, and J.-M. Lavest, "Monocular vision for mobile robot localization and autonomous navigation," International Journal of Computer Vision, vol. 74, no. 3, pp. 237-260, 2007.

[8] "Street View on Google Maps FAQ" http://maps.google.com/help/maps/streetview/faq.html, Google 2009

[9] Ioannis M. Rekleitis, “A Particle Filter Tutorial for Mobile Robot Localization”, Center for Intelligent Machines, McGill University. Thesis, 2002

[10] Dieter Fox, Wolfram Burgard, Sebastian Thrun, "Markov Localization for Robots in Dynamic Environments", Carnegie Mellon University, University of Freiburg. Thesis 1999

[11] W. Burgard, A. Derr, D. Fox, A. Cremers, "Integrating Global Position Estimation and Position Tracking for Mobile Robots: The Dynamic Markov Localization Approach", IEEE conference on Intelligent Robots and Systems, 1998

[12] Maria Isabel Ribeiro, "Kalman and Extended Kalman Filters: Concept, Derivation and Properties", Institute for Systems and Robotics in Portugal. Thesis, 2004

[13] Sonar, OpenCV, Accuracy and Laser Rangefinder on Wikipedia, March $16^{\text {th }}, 2008$

[14] "Cistern Exploration Project", http://users.csc.calpoly.edu/ cmclark/MaltaMapping/, Cal Poly San Luis Obispo, 2008 
[15] SICK - New Products, http://www.sickusa.com/gus/products/new/new_products/en, SICK, March $16^{\text {th }}, 2008$

[16] How Does GPS Work?, http://www.nasm.si.edu/gps/work.html, Smithsonian - National Air and Space Museum, 2005

[17] OpenCV on SourceForge, http://sourceforge.net/projects/opencvlibrary/, Source Forge, 2009

[18] "MATLAB", Wikipedia Coverage, http://en.wikipedia.org/wiki/MATLAB

[19] Image Processing Toolbox - Documentation, The MathWorks Help Desk, http://www.mathworks.com/access/helpdesk/help/toolbox/images/

[20] Image Correlation and Tracking, MATLAB Central - File Exchange,

http://www.mathworks.com/matlabcentral/fileexchange/12413

[21] Review of Logitech QuickCam Pro 5000, cNet Reviews, http://reviews.cnet.com/webcams/logitech-quickcam-pro-5000/4505-6502_7-

31529173.html?tag=mncol;lst; 1

[22] Conversion to Grey scale, Grokking the Gimp, http://gimp-

$\underline{\text { savvy.com/BOOK/index.html?node54.html }}$ 


\section{Appendix A: 'smooth_try3.m'}

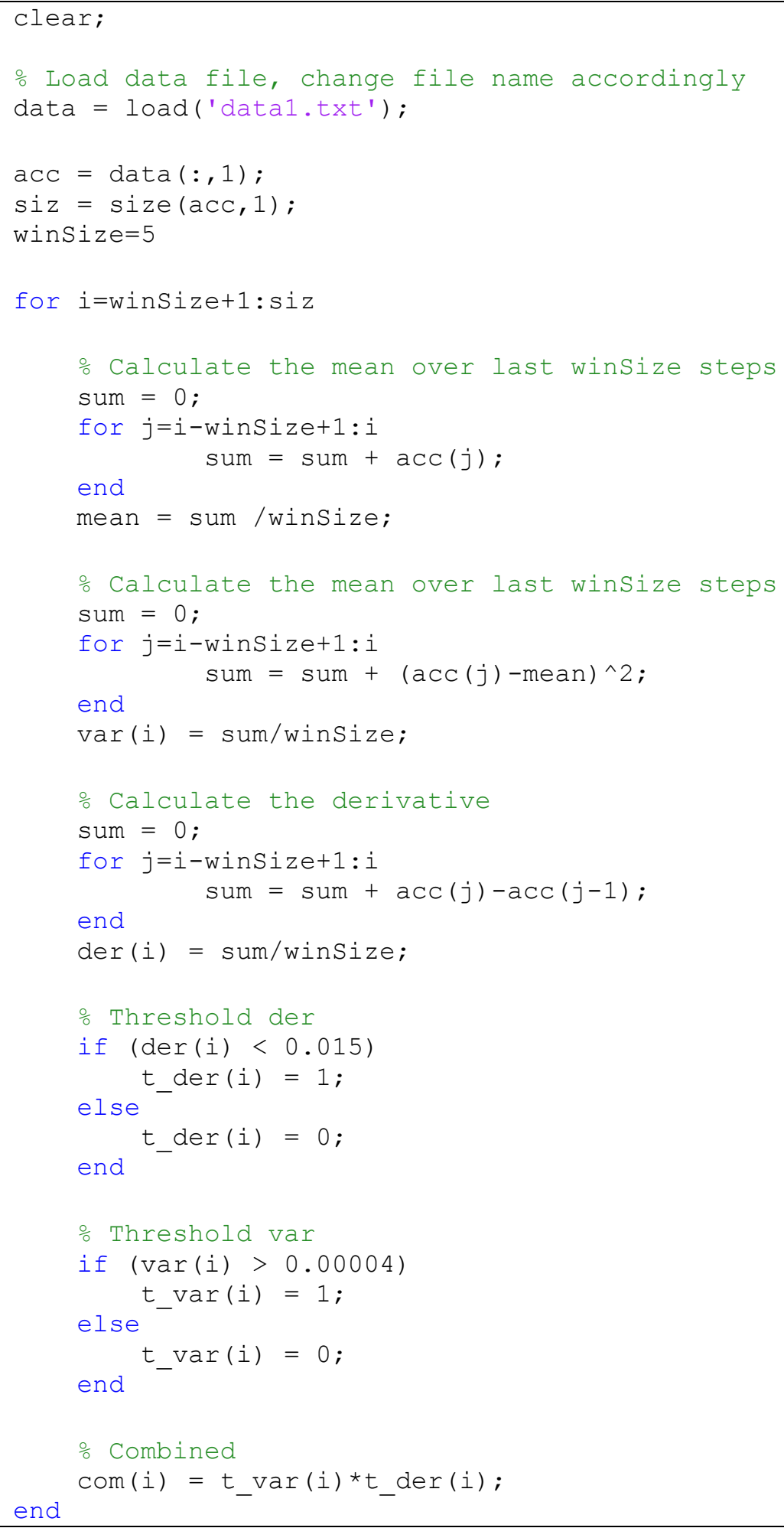




\section{Appendix B: 'IP_PF_0.m'}

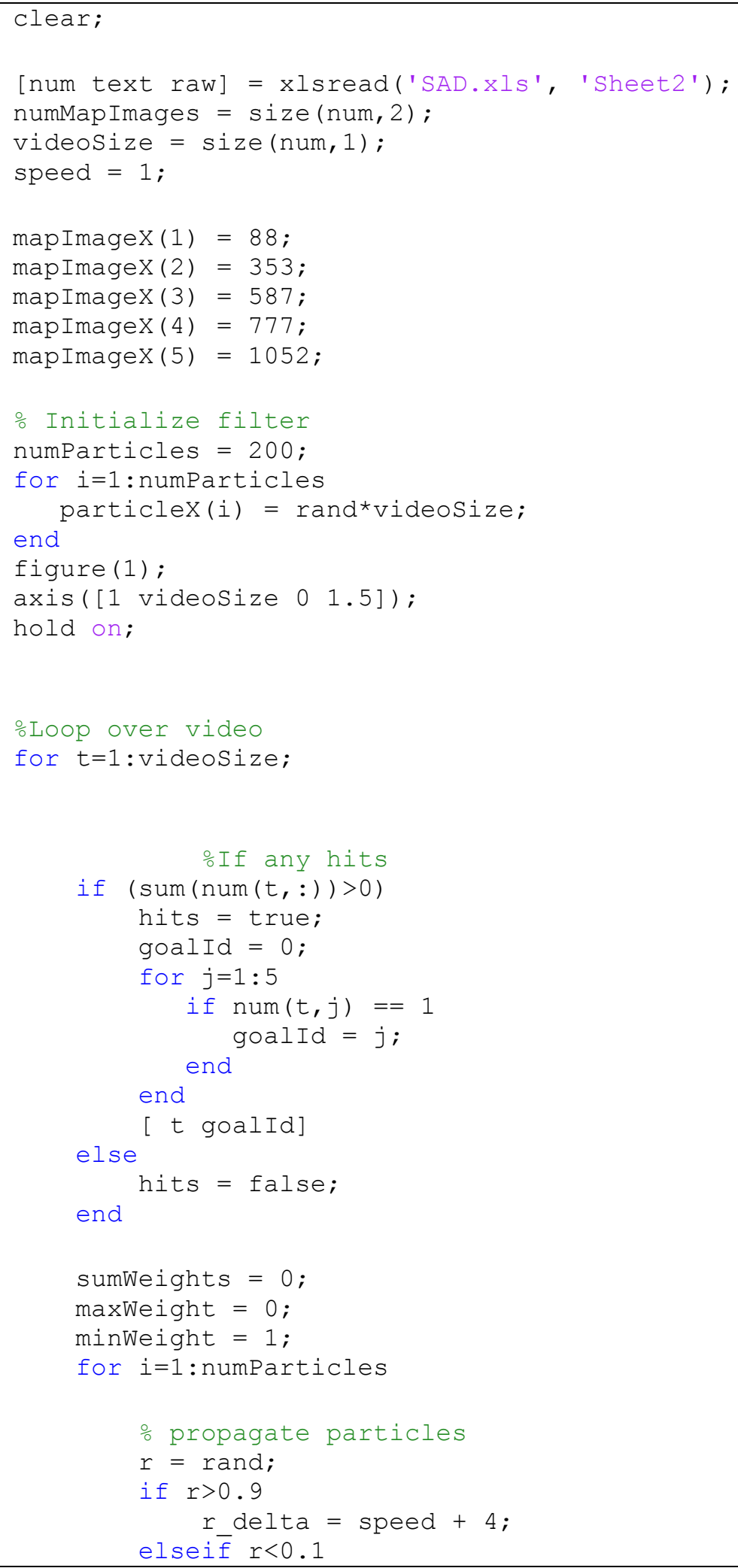




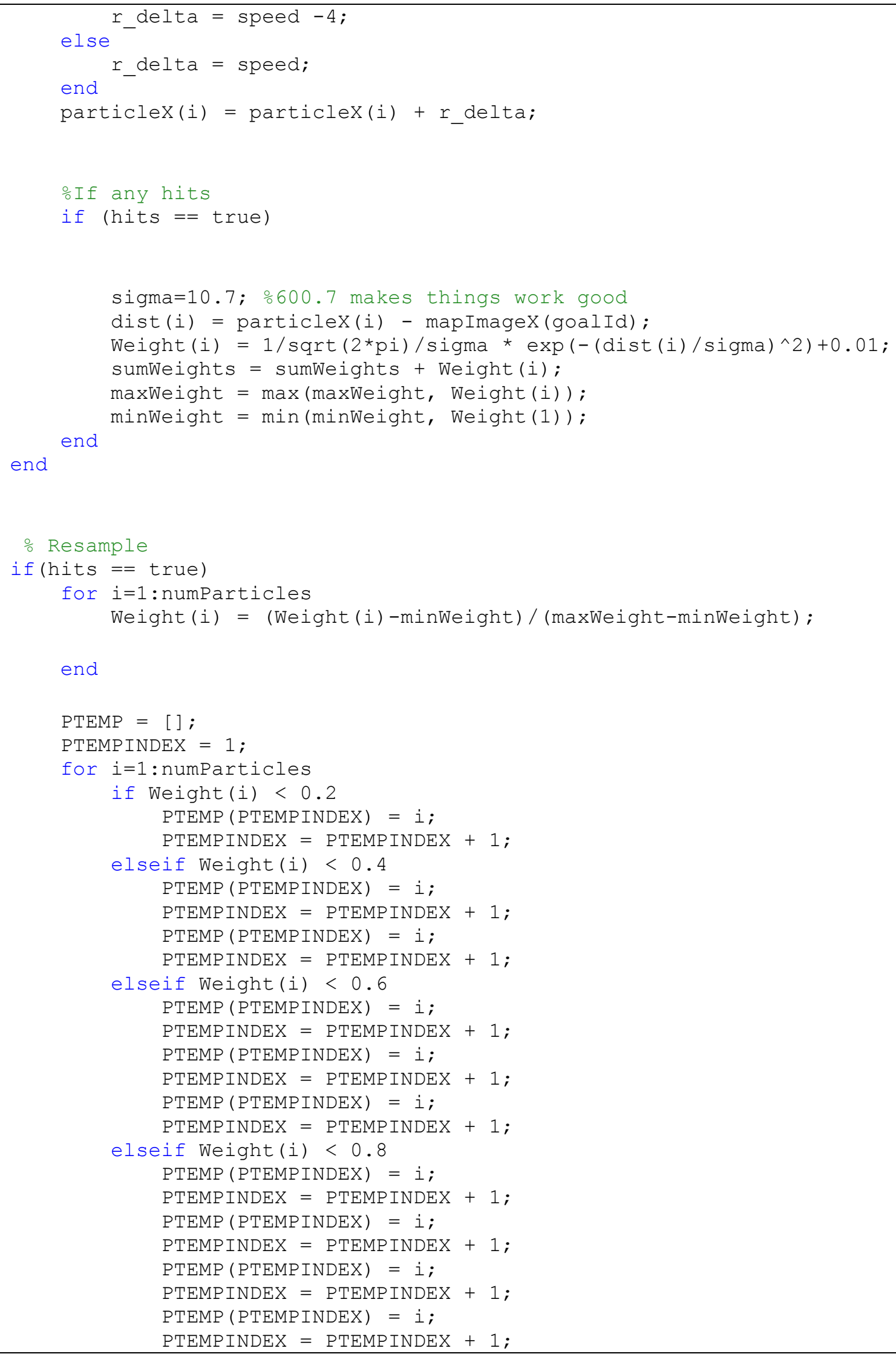




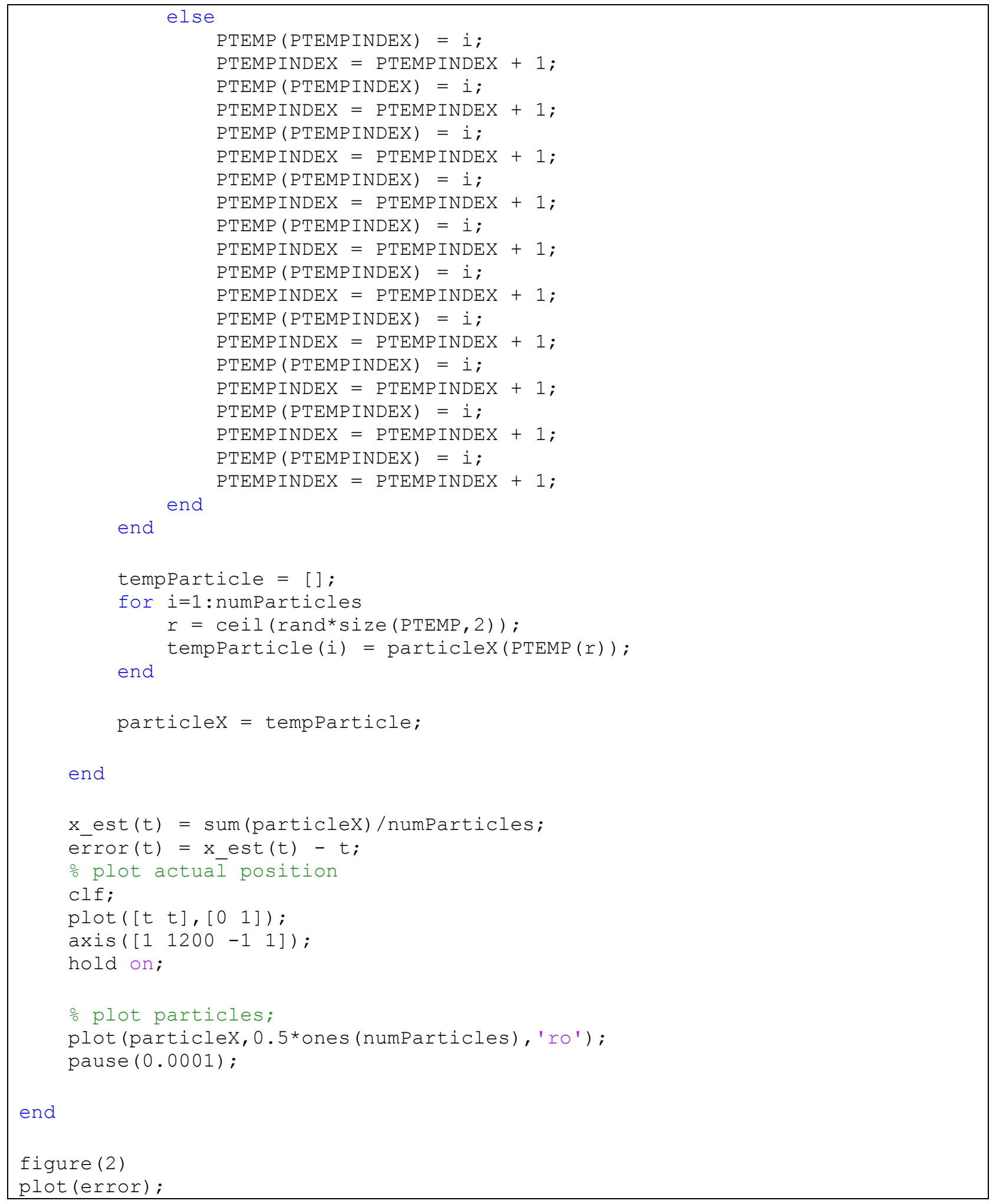

